\title{
Higgs as a Top-Mode Pseudo
}

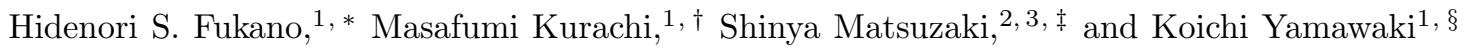 \\ 1 Kobayashi-Maskawa Institute for the Origin of Particles and the Universe (KMI) \\ Nagoya University, Nagoya 464-8602, Japan. \\ 2 Institute for Advanced Research, Nagoya University, Nagoya 464-8602, Japan. \\ ${ }^{3}$ Department of Physics, Nagoya University, Nagoya 464-8602, Japan.
}

\begin{abstract}
In the spirit of the top quark condensation, we propose a model which has a naturally light composite Higgs boson, "tHiggs" $\left(h_{t}^{0}\right)$, to be identified with the $126 \mathrm{GeV}$ Higgs discovered at the LHC. The tHiggs, a bound state of the top quark and its flavor (vector-like) partner, emerges as a pseudo Nambu-Goldstone boson (NGB), "Top-Mode Pseudo", together with the exact NGBs to be absorbed into the $W$ and $Z$ bosons as well as another (heavier) Top-Mode Pseudo (CP-odd composite scalar, $A_{t}^{0}$ ). Those five composite (exact/pseudo) NGBs are dynamically produced simultaneously by a single supercritical four-fermion interaction having $U(3) \times U(1)$ symmetry which includes the electroweak symmetry, where the vacuum is aligned by small explicit breaking term so as to break the symmetry down to a subgroup, $U(2) \times U(1)^{\prime}$, in a way not to retain the electroweak symmetry, in sharp contrast to the little Higgs models. The explicit breaking term for the vacuum alignment gives rise to a mass of the tHiggs, which is protected by the symmetry and hence naturally controlled against radiative corrections. Realistic top quark mass is easily realized similarly to the top-seesaw mechanism by introducing an extra (subcritical) four-fermion coupling which explicitly breaks the residual $U(2)^{\prime} \times U(1)^{\prime}$ symmetry with $U(2)^{\prime}$ being an extra symmetry beside the above $U(3)_{L} \times U(1)$. We present a phenomenological Lagrangian of the Top-Mode Pseudos along with the standard model particles, which will be useful for the study of the collider phenomenology. The coupling property of the tHiggs is shown to be consistent with the currently available data reported from the LHC. Several phenomenological consequences and constraints from experiments are also addressed.
\end{abstract}

\section{INTRODUCTION}

The ATLAS [1] and CMS collaborations 2] have discovered a new scalar particle at around $126 \mathrm{GeV}$ having the properties compatible with the Higgs boson in the Standard Model (SM). However, the origin of mass is still a mysterious, since we do not yet understand detailed features of the $126 \mathrm{GeV}$ Higgs, in particular the dynamical origin of the mass of the $126 \mathrm{GeV}$ Higgs itself which is just a free parameter in the SM.

A straightforward way to understand the dynamical origin of the Higgs boson in the explicit underlying theory beyond the SM is the walking technicolor having approximate scale invariance and large anomalous dimension $\gamma_{m} \simeq 1$, which predicts a technidilaton as a composite pseudo Nambu-Goldstone boson (NGB) of the approximate scale invariance [3, 4. It in fact was shown to be consistent with the current LHC data for the $126 \mathrm{GeV}$ Higgs [5, 6], and a recent lattice study [7] showed indication of such a very light flavor-singlet scalar meson as a candidate for the technidilaton in the walking technicolor. In order to accommodate a large top quark mass in the walking technicolor, however, we would need even larger anomalous dimension $\gamma_{m}>1$ for the techni-condensate relevant to the top mass, possibly realized by the additional strong four-fermion interaction (strong extended technicolor) 8 10. An alternative composite Higgs model based on such a strong four-fermion coupling is the top quark condensate model [11-16] with $\gamma_{m} \simeq 2$. Here we propose a variant of the top quark condensate model based on the strong four-fermion couplings, which yields a naturally light Higgs boson to be identified with the $126 \mathrm{GeV}$ Higgs boson at LHC.

Actually, among masses of the SM fermions, the top quark mass $\left(m_{t} \simeq 173 \mathrm{GeV}\right)$ is the only one roughly of the order of the electroweak symmetry breaking (EWSB) scale $\left(v_{\mathrm{EW}} \simeq 246 \mathrm{GeV}\right)$. Furthermore, the mass of the LHC Higgs boson $\left(m_{h} \simeq 126 \mathrm{GeV}\right)$ is also roughly of the order of the EWSB scale. This coincidence may imply that the top quark plays a crucial role for both the EWSB and the generation of the mass of the Higgs boson. In fact, before the top quark was discovered with the mass being this large, the top quark condensation (Top-Mode Standard Model; TMSM) was proposed [11, 12 to predict such a close relation among the top quark mass, the EWSB scale and the Higgs mass, based on the phase structure of the gauged Nambu-Jona-Lasinio (NJL) model [17, 18]. The four-fermion

\footnotetext{
* fukano@kmi.nagoya-u.ac.jp

${ }^{\dagger}$ kurachi@kmi.nagoya-u.ac.jp

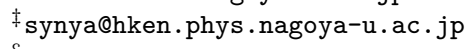

$\S$ yamawaki@kmi . nagoya-u.ac.jp
} 
interactions in the TMSM are written in the SM-gauge-invariant form [11, 12]:

$$
\mathcal{L}_{\mathrm{TMSM}}^{4 f}=G_{t}\left(\bar{q}_{L}^{i} t_{R}\right)\left(\bar{t}_{R} q_{L i}\right)+G_{b}\left(\bar{q}_{L}^{i} b_{R}\right)\left(\bar{b}_{R} q_{L_{i}}\right)+G_{t b}\left(\bar{q}_{L}^{i} q_{R}^{k}\right)\left(i \tau^{2}\right)^{i j}\left(i \tau^{2}\right)^{k l}\left(\bar{q}_{L}^{j} q_{R}^{l}\right)+\text { h.c. }
$$

where $q_{L(R)}=(t, b)_{L(R)}^{T}$ and $\tau^{2}$ is the second component of the Pauli matrices. It is straightforward to extend this to include all the three generations of the SM fermions [11, 12. The four-fermion interactions are arranged to trigger the top quark condensate, $\langle\bar{t} t\rangle \neq 0$, without other condensates such as the bottom condensate, $\langle\bar{b} b\rangle,\langle\bar{t} b\rangle, \cdots=0$, in such a way that

$$
G_{t}>G_{\text {crit }}>G_{b}, \cdots,
$$

where the critical coupling $G_{\text {crit }}$ is given as $G_{\text {crit }}=4 \pi^{2} /\left(N_{c} \Lambda^{2}\right)$, with $N_{c}$ being the number of QCD color and $\Lambda$ the cutoff scale of the theory, up to small corrections from the SM gauge interactions as implied by the phase structure of the gauged NJL model [17, 18. The solution of the gap equation indicates that the top quark mass can be much smaller than the cutoff scale $\Lambda, m_{t} \ll \Lambda$, by tuning the four-fermion coupling close to the critical coupling, $0<G_{t} / G_{\text {crit }}-1 \ll 1$. The TMSM produces three NGBs which are absorbed into the $W$ and $Z$ bosons when the electroweak gauge interactions are switched on, and predicts the top quark mass to be on the order of the EWSB scale, $v_{\mathrm{EW}} \simeq 246 \mathrm{GeV}$, through the Pagels-Stokar formula [19] for the decay constant $F_{\pi}\left(=v_{\mathrm{EW}}\right)$ of the NGBs, which are evaluated with the solution of the gap equation of the gauged NJL model.

However, the original TMSM has a few problems: i) Even if we assume the cutoff scale, $\Lambda$, is the Planck scale, the top quark mass is predicted to be $m_{t}=220-250 \mathrm{GeV}$ [11, 12, 16], which is somewhat larger than the experimental value $m_{t}^{\exp }=173 \mathrm{GeV}[20$. If we assume $\Lambda$ to be a few $\mathrm{TeV}$ to avoid excessive fine-tuning to reproduce the EWSB scale, we would face a disastrous situation where the top quark mass is too large: $m_{t} \sim 600 \mathrm{GeV}$ (top mass problem); ii) the TMSM predicts a Higgs boson as a $t \bar{t}$ bound state (the "top-Higgs boson", $H_{t}$ ) with mass in a range of $m_{t}<m_{H_{t}}<2 m_{t}$. Such a top-Higgs boson cannot be identified as the Higgs boson with the mass $\simeq 126 \mathrm{GeV}$ which was discovered at the LHC [1, 2] (Higgs mass problem).

The top-seesaw model 21 23 can solve the top mass problem. In the top-seesaw model, a new (vector-like) $S U(2)_{L^{-}}$ singlet quark (seesaw partner of the top quark) is introduced to mix with the $t_{R}$, which pulls the top quark mass down to the desired value $\simeq 173 \mathrm{GeV}$, so that the top mass problem is resolved. However, it turns out that the top-Higgs boson is still heavy, $m_{t}<m_{H_{t}}<2 m_{t}$, and therefore the Higgs mass problem still remains in the top-seesaw model.

The Higgs mass problem was recently resolved by the top-seesaw assisted technicolor model [24, 25], which is a hybrid version combining the top-seesaw model with technicolor. In this model a light top-Higgs boson with $m_{H_{t}}<m_{t}$ was realized by sharing the top quark mass with the technicolor sector. It was shown, however, that the coupling properties of the top-Higgs boson are quite different from those of the Higgs boson in the SM, and the model has currently been disfavored by the LHC data, most notably by the results on the diphoton decay channel [1, 2] and production cross section through the vector boson fusion process [26, 27.

In this paper, in the spirit of the top quark condensation, we propose a model which solves the Higgs mass problem in a natural way, while keeping the solution of the top mass problem by the top-seesaw mechanism. The light composite Higgs, what we call "tHiggs", emerges as one of the composite pseudo NGBs, dubbed "Top-Mode Pseudos", associated with the spontaneous breaking of an approximate global symmetry, which is triggered by strong (supercritical) four-fermion interactions.

The model is constructed from the third generation quarks in the SM $q=(t, b)$ and a (vector-like) $\chi$-quark which is a flavor partner of the top quark, and has the same SM charges as those of the right-handed top quark. The four-fermion interaction term takes the form,

$$
\mathcal{L}^{4 f}=G\left(\bar{\psi}_{L}^{i} \chi_{R}\right)\left(\bar{\chi}_{R} \psi_{L}^{i}\right),
$$

where $\psi_{L}^{i} \equiv\left(t_{L}, b_{L}, \chi_{L}\right)^{T i},(i=1,2,3)$. The four-fermion interaction in Eq. (3) possesses a global symmetry $U(3)_{L} \times$ $U(1)_{\chi_{R}}$. For the supercritical setting, $G>G_{\text {crit }}$, the symmetry is spontaneously broken down to $U(2)_{L} \times U(1)_{V}$ by the quark condensates $\left\langle\bar{\chi}_{R} t_{L}\right\rangle \neq 0$ and $\left\langle\bar{\chi}_{R} \chi_{L}\right\rangle \neq 0$, which are realized in the vacuum aligned with the additional explicit breaking terms mentioned below, while $\left\langle\bar{\chi}_{R} b_{L}\right\rangle$ is gauged away to $\left\langle\bar{\chi}_{R} b_{L}\right\rangle=0$ by the electroweak gauge symmetry when it is switched on. Note that the electroweak gauge symmetry is spontaneously broken when $U(3)_{L} \times U(1)_{\chi_{R}} \rightarrow$ $U(2)_{L} \times U(1)_{V}$, in sharp contrast to the little Higgs models. It should also be noted that the Lagrangian has $U(2)_{q_{R}}$ symmetry (see below Eq. (47)) of $\left(t_{R}, b_{R}\right)$ not broken by the condensate, which will not be explicitly mentioned unless becomes relevant.

Associated with this symmetry breaking, five NGBs emerge as bound states of the quarks. Besides those, a composite heavy Higgs boson corresponding to the $\sigma$ mode of the usual NJL model is also formed. Three of these NGBs will be eaten by the electroweak gauge bosons when the subgroup of the symmetry is gauged by the electroweak symmetry, while two of them remain as physical states. Those two NGBs, Top-Mode Pseudos, acquire their masses 
due to additional terms which explicitly break the $U(3)_{L} \times U(1)_{\chi_{R}}$ symmetry in such a way that the vacuum aligns to break the electroweak symmetry by $\left\langle\bar{\chi}_{R} t_{L}\right\rangle \neq 0$. One of them is a CP-even scalar (tHiggs, $h_{t}^{0}$ ), which is identified as the $126 \mathrm{GeV}$ Higgs boson discovered at the LHC, while the other is a heavy CP-odd scalar $\left(A_{t}^{0}\right)$, which is similar to the CP-odd Higgs in the two Higgs doublet models (there is an essential difference from the two-doublet Higgs model, though). We find a notable relation between masses of those Top-Mode Pseudos:

$$
m_{h_{t}^{0}}=m_{A_{t}^{0}} \sin \theta, \quad\left(\tan \theta=\frac{\left\langle\bar{\chi}_{R} t_{L}\right\rangle}{\left\langle\bar{\chi}_{R} \chi_{L}\right\rangle}\right),
$$

where the angle $\theta$ is related to the presence of the condensate, $\left\langle\bar{\chi}_{R} q_{L}\right\rangle \neq 0$, which causes the electroweak symmetry breaking.

It will be shown that the tHiggs couplings to the SM particles coincide with those of the SM Higgs boson in the limit $\sin \theta \rightarrow 0$ (where $v_{\mathrm{EW}} \simeq 246 \mathrm{GeV}$ is kept fixed). Even if the tHiggs coupling coincides with that of the SM Higgs, the virtue of our model is that the tHiggs $h_{t}^{0}$ is a bound state of the top quark and $\chi$-quark, and is natural in the sense that its mass is protected by the symmetry, in sharp contrast to the SM Higgs. One notable feature of our model is the prediction of the heavy CP-odd Higgs (without additional charged heavy Higgs in contrast to the two-doublet Higgs models), which will be tested in future collider experiments.

This paper is organized as follows: In Sec. III, we start with a simplified model based on four-fermion dynamics which has the (exact) global symmetry $U(3)_{L} \times U(1)_{\chi_{R}}$ to show that five NGBs emerge due to the spontaneous breaking of the global symmetry by the quark condensate generated by the supercritical four-fermion dynamics. Additional explicit breaking terms are then introduced to give masse to the two of the five NGBs (Top-Mode Pseudos; $h_{t}^{0}$ and $A_{t}^{0}$ ) and also to the top quark. We estimate the mass of the Top-Mode Pseudos based on the current algebra to find the mass formula Eq. (4). The interaction property of the tHiggs $\left(h_{t}^{0}\right)$ and the stability of the mass against the radiative corrections are addressed in comparison with the SM Higgs boson case. The extension of the model to incorporate masses of light fermions are also discussed in Sec. III. Several phenomenological constraints are given in Sec. III. Sec. IV] is devoted to the summary of this paper including some discussions. In appendix. A, we provide a straightforward derivation of the Top-Mode Pseudo masses by directly solving the bound state problem in the four-fermion dynamics based on the auxiliary field method. Appendix. B is devoted to some details of computations for one-loop corrections to the Top-Mode Pseudos arising from the top and its flavor partner, $t^{\prime}$-quark loops.

\section{MODEL}

In this section we propose a model based on four-fermion dynamics constructed from the top and bottom quarks $q=(t, b)$ with the flavor partner of top quark $(\chi)$. The model possesses an approximate global symmetry which is spontaneously broken by the quark condensates $\left\langle\bar{\chi}_{R} q_{L}\right\rangle \neq 0$ and $\left\langle\bar{\chi}_{R} \chi_{L}\right\rangle \neq 0$ generated by the four-fermion dynamics. Five NGBs emerge as bound states of the quarks associated with the spontaneous breaking of the global symmetry, in addition to a composite heavy Higgs boson which corresponds to the $\sigma$ mode in the usual NJL model. Two of the NGBs (what we call Top-Mode Pseudos $\left(h_{t}^{0}\right.$ and $\left.A_{t}^{0}\right)$ ) obtain their masses due to the introduction of additional four-fermion interactions which explicitly break the global symmetry, while other three remain massless to be eaten by the $W$ and $Z$ bosons once the electroweak charges are turned on. The mass of $h_{t}^{0}$ turns out to be protected by the global symmetry and the coupling property is shown to be consistent with the currently reported Higgs boson with mass around $\simeq 126 \mathrm{GeV}$. We will call $h_{t}^{0}$ tHiggs.

The present model, which is based on the strong four-fermion interactions with vector-like $\chi$-quark, can actually be viewed as a version of so-called top-seesaw model [21, 22. The crucial difference between existing top-seesaw models and the present model is that the present model makes it clear that the $126 \mathrm{GeV}$ Higgs exists as a pseudo NGB associated with the global symmetry breaking caused by four-fermion interactions. For the purpose of making this point clearer, in subsection II A we introduce a simplified model in which all the explicit breaking terms are turned off. In that simplified model, five NGBs which exist in the model are all massless. Then, in subsection IIB, we introduce explicit breaking terms into the Lagrangian to give masses to two of NGBs, Top Mode Pseudos $\left(h_{t}^{0}\right.$, $A_{t}^{0}$ ), which are identified as $126 \mathrm{GeV}$ Higgs boson and its CP-odd partner. Subsections II C and IID are devoted to explaining fermion masses, Yukawa interactions as well as the nature of the tHiggs.

\section{A. Structure of symmetry breaking}

Let us consider an NJL-like model constructed from the third generation quarks in the SM, $q=(t, b)$, and an $S U(2)_{L}$ singlet quark $(\chi)$. The left-handed quarks $q_{L}$ and $\chi_{L}$ form a flavor triplet $\psi_{L}^{i} \equiv\left(t_{L}, b_{L}, \chi_{L}\right)^{T i}(i=1,2,3)$ 
under the flavor $U(3)_{\psi_{L}}$ group, while the right-handed top and bottom quarks $q_{R}^{i} \equiv\left(t_{R}, b_{R}\right)^{T i}(i=1,2)$ and $\chi_{R}$ are a doublet and singlet under the $U(2)_{q_{R}}$ group, respectively. Turning off the SM gauge interactions momentarily, we thus write the Lagrangian having the global $U(3)_{\psi_{L}} \times U(2)_{q_{R}} \times U(1)_{\chi_{R}}$ symmetry:

$$
\mathcal{L}_{\text {kin. }}+\mathcal{L}^{4 f}=\bar{\psi}_{L} i \gamma^{\mu} \partial_{\mu} \psi_{L}+\bar{q}_{R} i \gamma^{\mu} \partial_{\mu} q_{R}+\bar{\chi}_{R} i \gamma^{\mu} \partial_{\mu} \chi_{R}+G\left(\bar{\psi}_{L}^{i} \chi_{R}\right)\left(\bar{\chi}_{R} \psi_{L}^{i}\right),
$$

where $G$ denotes the four-fermion coupling strength.

We can derive the gap equations for fermion dynamical masses $m_{t \chi}$ and $m_{\chi \chi}$ through the mean field relations $m_{t \chi}=-G\left\langle\bar{\chi}_{R} t_{L}\right\rangle$ and $m_{\chi \chi}=-G\left\langle\bar{\chi}_{R} \chi_{L}\right\rangle$ in the large $N_{c}$ limit: ${ }^{1}$

$$
\begin{aligned}
& m_{t \chi}=m_{t \chi} \frac{N_{c} G}{8 \pi^{2}}\left[\Lambda^{2}-\left(m_{t \chi}^{2}+m_{\chi \chi}^{2}\right) \ln \frac{\Lambda^{2}}{m_{t \chi}^{2}+m_{\chi \chi}^{2}}\right], \\
& m_{\chi \chi}=m_{\chi \chi} \frac{N_{c} G}{8 \pi^{2}}\left[\Lambda^{2}-\left(m_{t \chi}^{2}+m_{\chi \chi}^{2}\right) \ln \frac{\Lambda^{2}}{m_{t \chi}^{2}+m_{\chi \chi}^{2}}\right],
\end{aligned}
$$

where $\Lambda$ stands for the cutoff of the model which is to be of $\Lambda \gg \mathcal{O}(1) \mathrm{TeV}$. There exist nontrivial solutions $m_{t \chi} \neq 0$ and $m_{\chi \chi} \neq 0$ when the following criticality condition is satisfied:

$$
G>G_{\text {crit }}=\frac{8 \pi^{2}}{N_{c} \Lambda^{2}},
$$

under which we have the nonzero dynamical masses as well as the nonzero condensates,

$$
\left\langle\bar{\chi}_{R} q_{L}\right\rangle \neq 0, \quad\left\langle\bar{\chi}_{R} \chi_{L}\right\rangle \neq 0 .
$$

Note, however, that the two gap equations, Eqs. (6) and (7), cannot determine the ratio of two condensates: Those two gap equations with the criticality condition in Eq. (8) just lead to the nontrivial solution for the squared-sum of two masses, $\left(m_{t \chi}^{2}+m_{\chi \chi}^{2}\right) \neq 0$, so that the vacuum with $m_{t \chi} \neq 0$ is degenerate with that with $m_{t \chi}=0$. In order to lift the degeneracy for breaking the electroweak symmetry by $m_{t \chi} \neq 0$, we shall later introduce explicit breaking to align the vacuum, which also gives rise to the mass of two NGBs (Top-Mode Pseudos) out of five NGBs, with the rest three being exact NGBs to be absorbed into the $W$ and $Z$ bosons. Also note that the condensate $m_{b \chi} \neq 0$ can be gauged away when the model is gauged by the electroweak symmetry.

In order to make the structure of the symmetry breaking clearer, we may change the flavor basis of fermions by introducing an orthogonal rotation matrix $R$ :

$$
\tilde{\psi}_{L}=\left(\begin{array}{c}
\tilde{t}_{L} \\
\tilde{b}_{L} \\
\tilde{\chi}_{L}
\end{array}\right) \equiv R \cdot \psi_{L} \quad, \quad R=\left(\begin{array}{ccc}
\cos \theta & 0 & -\sin \theta \\
0 & 1 & 0 \\
\sin \theta & 0 & \cos \theta
\end{array}\right) \quad, \quad \tan \theta \equiv \frac{m_{t_{\chi}}}{m_{\chi \chi}}=\frac{\left\langle\bar{\chi}_{R} t_{L}\right\rangle}{\left\langle\bar{\chi}_{R} \chi_{L}\right\rangle} .
$$

The two gap equations, Eqs. (6) and (7), are then reduced to a single gap equation,

$$
1=\frac{N_{c} G}{8 \pi^{2}}\left[\Lambda^{2}-m_{\tilde{\chi} \chi}^{2} \ln \frac{\Lambda^{2}}{m_{\tilde{\chi} \chi}^{2}}\right],
$$

with

$$
m_{\tilde{\chi} \chi}^{2} \equiv m_{t \chi}^{2}+m_{\chi \chi}^{2} \neq 0
$$

Accordingly, the associated two condensates in Eq. 9 are reduced to a single nonzero condensate on the basis of $\tilde{\psi}_{L}$ :

$$
\left\langle\bar{\chi}_{R} \tilde{\chi}_{L}\right\rangle \neq 0 \text {. }
$$

We thus see that, with the criticality condition in Eq.8 satisfied, the four-fermion dynamics triggers the following global symmetry breaking pattern:

$$
U(3)_{\tilde{\psi}_{L}} \times U(1)_{\chi_{R}} \rightarrow U(2)_{\tilde{q}_{L}} \times U(1)_{V=\tilde{\chi}_{L}+\chi_{R}}
$$
1 We have put $m_{b \chi}=0$, by gauging it away by the electroweak gauge symmetry. Otherwise $\left(m_{t \chi}^{2}+m_{\chi \chi}^{2}\right)$ in Eqs. 6 and $\sqrt{7}$ should read
$\left(m_{t \chi}^{2}+m_{b \chi}^{2}+m_{\chi \chi}^{2}\right)$ because of $U(3)_{\psi_{L}}$ symmetry, with $m_{b \chi}$ also satisfying the same type of gap equation. 
The broken currents associated with this symmetry breaking are found to be

$$
\begin{aligned}
J_{3 L}^{4, \mu} & =\overline{\tilde{\psi}}_{L} \gamma^{\mu} \lambda^{4} \tilde{\psi}_{L} \\
& =\overline{\tilde{t}}_{L} \gamma^{\mu} \tilde{\chi}_{L}+\overline{\tilde{\chi}}_{L} \gamma^{\mu} \tilde{t}_{L} \\
& =\left(\bar{t}_{L} \gamma^{\mu} t_{L}+\bar{\chi}_{L} \gamma^{\mu} \chi_{L}\right) \sin 2 \theta+\left(\bar{t}_{L} \gamma^{\mu} \chi_{L}+\bar{\chi}_{L} \gamma^{\mu} t_{L}\right) \cos 2 \theta \\
J_{3 L}^{5, \mu} & =\overline{\tilde{\psi}}_{L} \gamma^{\mu} \lambda^{5} \tilde{\psi}_{L} \\
& =i\left[-\overline{\tilde{t}}_{L} \gamma^{\mu} \tilde{\chi}_{L}+\overline{\tilde{\chi}}_{L} \gamma^{\mu} \tilde{t}_{L}\right] \\
& =-i\left(\bar{t}_{L} \gamma^{\mu} \chi_{L}-\bar{\chi}_{L} \gamma^{\mu} t_{L}\right) \\
J_{3 L}^{6, \mu} & =\overline{\tilde{\psi}}_{L} \gamma^{\mu} \lambda^{6} \tilde{\psi}_{L} \\
& =\overline{\tilde{b}}_{L} \gamma^{\mu} \tilde{\chi}_{L}+\overline{\tilde{\chi}}_{L} \gamma^{\mu} \tilde{b}_{L} \\
& =\left(\bar{b}_{L} \gamma^{\mu} t_{L}+\bar{t}_{L} \gamma^{\mu} b_{L}\right) \sin \theta+\left(\bar{b}_{L} \gamma^{\mu} \chi_{L}+\bar{\chi}_{L} \gamma^{\mu} b_{L}\right) \cos \theta \\
J_{3 L}^{7, \mu} & =\overline{\tilde{\psi}}_{L} \gamma^{\mu} \lambda^{7} \tilde{\psi}_{L} \\
& =i\left[-\overline{\tilde{b}}_{L} \gamma^{\mu} \tilde{\chi}_{L}+\overline{\tilde{\chi}}_{L} \gamma^{\mu} \tilde{b}_{L}\right] \\
& =-i\left(\bar{b}_{L} \gamma^{\mu} t_{L}-\bar{t}_{L} \gamma^{\mu} b_{L}\right) \sin \theta-i\left(\bar{b}_{L} \gamma^{\mu} \chi_{L}-\bar{\chi}_{L} \gamma^{\mu} b_{L}\right) \cos \theta,
\end{aligned}
$$

and

$$
\begin{aligned}
J_{A}^{\mu} & \equiv \frac{1}{4}\left(J_{1 R}^{\mu}-\frac{1}{\sqrt{6}} J_{3 L}^{0, \mu}+\frac{1}{\sqrt{3}} J_{3 L}^{8, \mu}\right) \\
& =\frac{1}{4}\left(\bar{\chi}_{R} \gamma^{\mu} \chi_{R}-\overline{\tilde{\chi}}_{L} \gamma^{\mu} \tilde{\chi}_{L}\right) \\
& =\frac{1}{4}\left[\bar{\chi}_{R} \gamma^{\mu} \chi_{R}-\bar{t}_{L} \gamma^{\mu} t_{L} \sin ^{2} \theta-\bar{\chi}_{L} \gamma^{\mu} \chi_{L} \cos ^{2} \theta-\left(\bar{t}_{L} \gamma^{\mu} \chi_{L}+\bar{\chi}_{L} \gamma^{\mu} t_{L}\right) \sin \theta \cos \theta\right]
\end{aligned}
$$

where the Gell-Mann matrices $\lambda^{a}(a=1, \cdots, 8)$ are normalized as $\operatorname{tr}\left[\lambda^{a} \lambda^{b}\right]=2 \delta^{a b}$, and $\lambda^{0}=\sqrt{2 / 3} 1_{3 \times 3}$. The associated NGBs emerge with the decay constant $f$ as

$$
\left\langle 0\left|J_{\mu}^{a}(x)\right| \pi_{t}^{b}(p)\right\rangle=-i f \delta^{a b} p_{\mu} e^{-i p \cdot x}, \quad a, b=4,5,6,7, A .
$$

The decay constant $f$ is calculated through the Pagels-Stokar formula [19]:

$$
f^{2}=\frac{N_{c}}{8 \pi^{2}} m_{\tilde{\chi} \chi}^{2} \ln \frac{\Lambda^{2}}{m_{\tilde{\chi} \chi}^{2}} .
$$

The five NGBs $\left(\pi_{t}^{a}\right)$ can be expressed as composite fields (interpolating fields) made of the fermion bilinears on the basis of $\left(\tilde{\psi}_{L}, \chi_{R}\right)$ or $\left(\psi_{L}, \chi_{R}\right)$ :

$$
\begin{aligned}
\pi_{t}^{4} & \sim \bar{\chi}_{R} \tilde{t}_{L}-\overline{\tilde{t}}_{L} \chi_{R} \\
& =\left(\bar{\chi}_{R} t_{L}-\bar{t}_{L} \chi_{R}\right) \cos \theta-\left(\bar{\chi}_{R} \chi_{L}-\bar{\chi}_{L} \chi_{R}\right) \sin \theta \\
\pi_{t}^{5} & \sim-i\left(\bar{\chi}_{R} \tilde{t}_{L}+\tilde{\tilde{t}}_{L} \chi_{R}\right) \\
& =-i\left(\bar{\chi}_{R} t_{L}+\bar{t}_{L} \chi_{R}\right) \cos \theta+i\left(\bar{\chi}_{R} \chi_{L}+\bar{\chi}_{L} \chi_{R}\right) \sin \theta \\
\pi_{t}^{6}+i \pi_{t}^{7} & \sim\left(\bar{\chi}_{R} \tilde{b}_{L}-\overline{\tilde{b}}_{L} \chi_{R}\right)+\left(\bar{\chi}_{R} \tilde{b}_{L}+\overline{\tilde{b}}_{L} \chi_{R}\right) \\
& =2 \bar{\chi}_{R} b_{L} \\
\pi_{t}^{6}-i \pi_{t}^{7} & \sim\left(\bar{\chi}_{R} \tilde{b}_{L}-\overline{\tilde{b}}_{L} \chi_{R}\right)-\left(\bar{\chi}_{R} \tilde{b}_{L}+\overline{\tilde{b}}_{L} \chi_{R}\right) \\
& =-2 \bar{b}_{L} \chi_{R}, \\
\pi_{t}^{A} & \sim \bar{\chi}_{R} \tilde{\chi}_{L}-\overline{\tilde{\chi}}_{L} \chi_{R} \\
& =\left(\bar{\chi}_{R} t_{L}-\bar{t}_{L} \chi_{R}\right) \sin \theta+\left(\bar{\chi}_{R} \chi_{L}-\bar{\chi}_{L} \chi_{R}\right) \cos \theta .
\end{aligned}
$$


Besides these composite NGBs, there exists a composite scalar $\left(H_{t}^{0}\right)$ corresponding to the $\sigma$ mode in the usual NJL model,

$$
\begin{aligned}
H_{t}^{0} & \sim \bar{\chi}_{R} \tilde{\chi}_{L}+\overline{\tilde{\chi}}_{L} \chi_{R} \\
& =\left(\bar{\chi}_{R} t_{L}+\bar{t}_{L} \chi_{R}\right) \sin \theta+\left(\bar{\chi}_{R} \chi_{L}+\bar{\chi}_{L} \chi_{R}\right) \cos \theta
\end{aligned}
$$

with the mass

$$
m_{H_{t}^{0}}^{2}=4 m_{\tilde{\chi} \chi}^{2}=4\left(m_{t \chi}^{2}+m_{\chi \chi}^{2}\right) .
$$

The $H_{t}^{0}$ will be regarded as a heavy Higgs boson with the mass of $\mathcal{O}(1) \mathrm{TeV}$, not the light Higgs boson at around 126 $\mathrm{GeV}$.

With the electroweak gauge interactions turned on, the $W$ and $Z$ bosons turn out to couple to the broken currents $\left(J_{3 L}^{6, \mu} \mp i J_{3 L}^{\mu, 7}\right)$ and $\left(J_{3 L}^{\mu, 4} \cos \theta+J_{3 L}^{\mu, A} \sin \theta\right)$, respectively. The corresponding would-be NGBs $\left(w_{t}^{ \pm}, z_{t}^{0}\right)$ eaten by the electroweak gauge bosons are then found to be

$$
\begin{aligned}
z_{t}^{0} & \equiv \pi_{t}^{4} \cos \theta+\pi_{t}^{A} \sin \theta \\
& \sim \bar{\chi}_{R} t_{L}-\bar{t}_{L} \chi_{R}, \\
w_{t}^{-} & \equiv \frac{1}{\sqrt{2}}\left(\pi_{t}^{6}+i \pi_{t}^{7}\right) \\
& \sim \sqrt{2} \bar{\chi}_{R} b_{L}, \\
w_{t}^{+} & \equiv \frac{1}{\sqrt{2}}\left(\pi_{t}^{6}-i \pi_{t}^{7}\right) \\
& \sim-\sqrt{2} \bar{b}_{L} \chi_{R} .
\end{aligned}
$$

On the other hand, the following two NGBs remain as physical states:

$$
\begin{aligned}
h_{t}^{0} & \equiv \pi_{t}^{5} \\
& \sim-i\left(\bar{\chi}_{R} \tilde{t}_{L}+\overline{\tilde{t}}_{L} \chi_{R}\right) \\
& =-i\left(\bar{\chi}_{R} t_{L}+\bar{t}_{L} \chi_{R}\right) \cos \theta+i\left(\bar{\chi}_{R} \chi_{L}+\bar{\chi}_{L} \chi_{R}\right) \sin \theta, \\
A_{t}^{0} & \equiv-\pi_{t}^{4} \sin \theta+\pi_{t}^{A} \cos \theta \\
& \sim \bar{\chi}_{R} \chi_{L}-\bar{\chi}_{L} \chi_{R} .
\end{aligned}
$$

The correspondence between the broken currents and NGBs along with the CP transformation property is summarized in TableI. The two massless NGBs $\left(h_{t}^{0}, A_{t}^{0}\right)$ will become pseudo NGBs, called "Top-Mode Pseudos", obtaining their masses once explicit breaking effects are introduced (see Sec. IIB). We will identify the CP-even Top-Mode Pseudo, $h_{t}^{0}$, as the $126 \mathrm{GeV}$ Higgs, called tHiggs.

\begin{tabular}{|c|c|c|}
\hline Broken current & corresponding NGB & CP-property \\
\hline$J_{3 L}^{4, \mu}$ & $\pi_{t}^{4}=z_{t}^{0} \cos \theta-A_{t}^{0} \sin \theta$ & odd \\
\hline$J_{3 L}^{5, \mu}$ & $\pi_{t}^{5}=h_{t}^{0}$ & even \\
\hline$J_{3 L}^{6, \mu} \pm i J_{3 L}^{7, \mu}$ & $\pi_{t}^{6} \pm i \pi_{t}^{7}=\sqrt{2} w_{t}^{\mp}$ & - \\
\hline$J_{A}^{\mu}$ & $\pi_{t}^{A}=z_{t}^{0} \sin \theta+A_{t}^{0} \cos \theta$ & odd \\
\hline
\end{tabular}

TABLE I: The list of the broken currents and NGBs associated with the spontaneous symmetry breaking in Eq.(14). $w_{t}^{ \pm}$and $z_{t}^{0}$ are eaten by the $W^{ \pm}$and $Z$ bosons once the electroweak gauges are turned on, while the remaining two $A_{t}^{0}$ and $h_{t}^{0}$ become pseudo NGBs (Top-Mode Pseudos) by explicit breaking effects (see Eqs.40] and 441).

We may integrate out the heavy Higgs boson $H_{t}^{0}$ (with mass of $\mathcal{O}(1) \mathrm{TeV}$ ) to construct the low-energy effective theory governed by the five NGBs $\left(\pi_{t}^{a}\right)$ described by a nonlinear sigma model based on the coset space,

$$
\frac{\mathcal{G}}{\mathcal{H}}=\frac{U(3)_{\tilde{\psi}_{L}} \times U(1)_{\chi_{R}}}{U(2)_{\tilde{\psi}_{L}} \times U(1)_{V=\chi_{L}+\chi_{R}}} .
$$


For this purpose we introduce representatives $\left(\xi_{L, R}\right)$ of the $\mathcal{G} / \mathcal{H}$ which are parameterized by NGB fields as

$$
\xi_{L}=\exp \left[-\frac{i}{f}\left(\sum_{a=4,5,6,7} \pi_{t}^{a} \lambda^{a}+\frac{\pi_{t}^{A}}{2 \sqrt{2}} \lambda^{A}\right)\right] \quad, \quad \xi_{R}=\exp \left[\frac{i}{f} \frac{\pi_{t}^{A}}{2 \sqrt{2}} \lambda^{A}\right]
$$

where

$$
\lambda^{A}=\left(\begin{array}{ccc}
0 & 0 & 0 \\
0 & 0 & 0 \\
0 & 0 & \sqrt{2}
\end{array}\right)
$$

We further introduce the "chiral" field $U$,

$$
U=\xi_{L}^{\dagger} \cdot \Sigma \cdot \xi_{R} \quad \text { with } \quad \Sigma=\frac{1}{\sqrt{2}} \lambda^{A}
$$

The transformation properties of $\xi_{L, R}$ and $U$ under $\mathcal{G}$ are given by

$$
\xi_{L} \rightarrow h\left(\pi_{t}, \tilde{g}\right) \cdot \xi_{L} \cdot g_{3 L}^{\dagger} \quad, \quad \xi_{R} \rightarrow h\left(\pi_{t}, \tilde{g}\right) \cdot \xi_{R} \cdot g_{1 R}^{\dagger} \quad, \quad U \rightarrow g_{3 L} \cdot U \cdot g_{1 R}^{\dagger}
$$

where $\tilde{g}=\left\{g_{3 \tilde{3 L}}, g_{1 R}\right\}, g_{3 L} \in U(3)_{\tilde{\psi}_{L}}, g_{1 R} \in U(1)_{\chi_{R}}$ and $h\left(\pi_{t}, \tilde{g}\right) \in \mathcal{H}$. Thus we find the $\mathcal{G}$-invariant Lagrangian written in terms of the NGBs to the lowest order of derivatives of $\mathcal{O}\left(p^{2}\right)$ :

$$
\mathcal{L}_{\mathrm{NL} \sigma \mathrm{M}}=\frac{f^{2}}{2} \operatorname{tr}\left[\partial_{\mu} U^{\dagger} \partial^{\mu} U\right]
$$

When the electroweak symmetry turned on, the covariant derivative acting on $U$ is given by

$$
D_{\mu} U \equiv R\left[\partial_{\mu}-i g \sum_{a=1}^{3} W_{\mu}^{a}\left(\begin{array}{cc|c}
\tau^{a} / 2 & 0 \\
\hline 0 & 0 & 0
\end{array}\right)+i g^{\prime} B_{\mu}\left(\begin{array}{ccc}
1 / 2 & 0 & 0 \\
0 & 1 / 2 & 0 \\
0 & 0 & 0
\end{array}\right)\right] R^{T} \cdot U,
$$

where $W_{\mu}$ and $B_{\mu}$ are the $S U(2)_{L}$ and $U(1)_{Y}$ gauge boson fields with the gauge couplings $g$ and $g^{\prime}$. Then the Lagrangian Eq. 26 ) is changed to the covariant form:

$$
\mathcal{L}_{\mathrm{NL} \sigma \mathrm{M}}=\frac{f^{2}}{2} \operatorname{tr}\left[D_{\mu} U^{\dagger} D^{\mu} U\right]
$$

From this one can read off the $W$ an $Z$ boson masses as

$$
m_{W}^{2}=\frac{1}{4} g^{2} f^{2} \sin ^{2} \theta \quad, \quad m_{Z}^{2}=\frac{1}{4}\left(g^{2}+g^{\prime 2}\right) f^{2} \sin ^{2} \theta,
$$

which lead to

$$
\begin{aligned}
v_{\mathrm{EW}}^{2} & =f^{2} \sin ^{2} \theta \\
& =\frac{N_{c}}{8 \pi^{2}} m_{t \chi}^{2} \ln \frac{\Lambda^{2}}{m_{t \chi}^{2}+m_{\chi \chi}^{2}} \\
& \simeq(246 \mathrm{GeV})^{2},
\end{aligned}
$$

where use has been made of Eq. 21]. Thus imposing the EWSB scale $v_{\mathrm{EW}}$ gives a nontrivial relation between $m_{t \chi}$ and $m_{\chi \chi}$.

Note that switching on the electroweak gauge interaction explicitly breaks the $U(3)_{\tilde{\psi}_{L}} \times U(1)_{\chi_{R}}$ symmetry. Such explicit breaking effects would generate masses to the NGBs at the loop level, as a part of the $1 / N_{c}$ sub-leading effect, which are, however, negligibly small since the size of effects is suppressed by the small electroweak gauge coupling, as will be discussed later (see the discussion below Eq. (80)).

As we mentioned earlier the criticality $G>G_{\text {crit }}$ implies $\left\langle\bar{\chi}_{R} t_{L}\right\rangle^{2}+\left\langle\bar{\chi}_{R} \chi_{L}\right\rangle^{2} \neq 0$, but not necessarily $\left\langle\bar{\chi}_{R} t_{L}\right\rangle \neq 0$ which is responsible for the electroweak symmetry breaking. The electroweak gauge interaction itself can contribute to lifting the degeneracy between the vacuum with $\left\langle\bar{\chi}_{R} t_{L}\right\rangle=0$ and that with $\left\langle\bar{\chi}_{R} t_{L}\right\rangle \neq 0$ in principle by some extreme fine tuning of the critical coupling of the gauged NJL model [17, 18.

More natural way will be to introduce extra effective four-fermion interactions to explicitly break the $U(3)_{\tilde{\psi}_{L}} \times$ $U(1)_{\chi_{R}}$ symmetry, which can align the vacuum to have $\left\langle\bar{\chi}_{R} t_{L}\right\rangle \neq 0$ and simultaneously give the mass of the tHiggs on the right amount. Such an explicit breaking may be induced from a strong $U(1)$ gauge interaction distinguishing $\left\langle\bar{\chi}_{R} t_{L}\right\rangle$ from $\left\langle\bar{\chi}_{R} \chi_{L}\right\rangle$. This we perform in the next subsection. 


\section{B. Top-Mode Pseudos}

Here we incorporate explicit breaking terms into the Lagrangian Eq. (5) to give masses to the Top-Mode Pseudos $\left(h_{t}^{0}, A_{t}^{0}\right)$ :

$$
\mathcal{L}_{\text {kin. }}+\mathcal{L}^{4 f}+\mathcal{L}^{h}
$$

where

$$
\mathcal{L}^{h}=-\left[\Delta_{\chi \chi} \bar{\chi}_{R} \chi_{L}+\text { h.c. }\right]-G^{\prime}\left(\bar{\chi}_{L} \chi_{R}\right)\left(\bar{\chi}_{R} \chi_{L}\right)
$$

Similarly to Eqs. (6) and (7), we derive the gap equations for fermion dynamical masses $m_{t \chi}$ and $m_{\chi \chi}$ :

$$
\begin{aligned}
& m_{t \chi}=m_{t \chi} \frac{N_{c} G}{8 \pi^{2}}\left[\Lambda^{2}-m_{\tilde{\chi} \chi}^{2} \ln \frac{\Lambda^{2}}{m_{\tilde{\chi} \chi}^{2}}\right], \\
& m_{\chi \chi}=\Delta_{\chi \chi}+m_{\chi \chi} \frac{N_{c}\left(G-G^{\prime}\right)}{8 \pi^{2}}\left[\Lambda^{2}-m_{\tilde{\chi} \chi}^{2} \ln \frac{\Lambda^{2}}{m_{\tilde{\chi} \chi}^{2}}\right],
\end{aligned}
$$

where $m_{\tilde{\chi} \chi}^{2}=m_{t \chi}^{2}+m_{\chi \chi}^{2}$. Note that the nonzero $\Delta_{\chi \chi}$ and $G^{\prime}$ allow to determine the ratio of two dynamical masses $m_{t \chi}$ and $m_{\chi \chi}$, i.e. $\tan \theta=m_{t \chi} / m_{\chi \chi}$, in contrast to the previous gap equations, Eqs. (6) and (7), which only determine the squared-sum of two, $m_{t \chi}^{2}+m_{\chi \chi}^{2}$.

Furthermore, it turns out that these $\Delta_{\chi \chi}$ and $G^{\prime}$ terms do not affect the criticality of the four-fermion dynamics at all: Assuming $m_{t \chi} \neq 0$ and $m_{\chi \chi} \neq 0$, we find that the following relation is required so as to keep the self consistency in the gap equations:

$$
\Delta_{\chi \chi}=\frac{G^{\prime}}{G} m_{\chi \chi}=-G^{\prime}\left\langle\bar{\chi}_{R} \chi_{L}\right\rangle .
$$

By taking this into account, the two gap equations, Eqs. 32 and (33), are reduced to a single one,

$$
1=\frac{N_{c} G}{8 \pi^{2}}\left[\Lambda^{2}-m_{\tilde{\chi} \chi}^{2} \ln \frac{\Lambda^{2}}{m_{\tilde{\chi} \chi}^{2}}\right] .
$$

Thus the presence of the nontrivial solution is controlled solely by $G>G_{\text {crit }}$, which is the same criticality condition as in Eq.(8). The Lagrangian Eq. (31) is also rewritten as

$$
\mathcal{L}^{h}=-G^{\prime}\left(\bar{\chi}_{L} \chi_{R}-\left\langle\bar{\chi}_{L} \chi_{R}\right\rangle\right)\left(\bar{\chi}_{R} \chi_{L}-\left\langle\bar{\chi}_{R} \chi_{L}\right\rangle\right)
$$

This implies that the explicit breaking effects can also be expressed only by the four-fermion interaction. We can thus approximately keep the global $U(3)_{\tilde{\psi}_{L}} \times U(1)_{\chi_{R}}$ invariance by taking $G^{\prime}$ perturbatively to be

$$
0<\frac{G^{\prime}}{G} \ll 1 \quad \text { and } \quad G_{\text {crit }}<G .
$$

We have explicitly checked that in the presence of this explicit breaking the vacuum with $m_{t \chi} \neq 0$ is preferred to that with $m_{t \chi}=0$ in the phenomenologically interesting parameter space to be discussed later.

As in Sec. II A the global $U(3)_{\tilde{\psi}_{L}} \times U(1)_{\chi_{R}}$ symmetry is spontaneously broken down to $U(2)_{\tilde{\psi}_{L}} \times U(1)_{V=\tilde{\chi}_{L}+\chi_{R}}$ due to the four-fermion interaction in $\mathcal{L}^{4 f}$, resulting in the presence of five $\operatorname{NGBs} \pi^{a}(a=4,5,6,7, A)$. Then the explicit breaking terms in $\mathcal{L}^{h}$ force the vacuum to choose a specific direction, $\left\langle\bar{\chi}_{R} \tilde{\chi}_{L}\right\rangle \neq 0$, and give masses to some of the NGBs. Note that the $\mathcal{L}^{h}$ term is invariant under the chiral transformation associated with the broken currents $\left(J_{3 L}^{6, \mu} \pm i J_{3 L}^{7, \mu}\right)$ and $\left(J_{3 L}^{4, \mu} \cos \theta+J_{3 L}^{A, \mu} \sin \theta\right)$, but not for $J_{\mu 3 L}^{5}$ and $\left(-J_{3 L}^{4, \mu} \sin \theta+J_{3 L}^{A, \mu} \cos \theta\right)$. Hence $\mathcal{L}^{h}$ term gives masses only to the NGBs associate with latter two, i.e., the Top-Mode Pseudos $A_{t}^{0}$ and $h_{t}^{0}$.

Estimation of the masses of the pseudo NGBs can be done by the traditional approach based on the current algebra [28]:

$$
m_{a b}^{2}=\frac{1}{f^{2}}\left\langle 0\left|\left[i Q^{a},\left[i Q^{b},-\mathcal{L}^{h}\right]\right]\right| 0\right\rangle
$$


where $Q^{a}$ is the Noether's charge associated with the broken currents Eqs. 115$),(16),(17),(18)$ and $(19)$, and $f$ is given by Eq.(21). From Eq.(38), together with the gap equations, Eqs.(32), (33) and (34), we obtain

$$
\begin{aligned}
m_{z_{t}^{0}}^{2} & =m_{w_{t}^{ \pm}}^{2}=0 \\
m_{A_{t}^{0}}^{2} & =\frac{2\left\langle\bar{\chi}_{R} \tilde{\chi}_{L}\right\rangle\left\langle\bar{\chi}_{R} \chi_{L}\right\rangle}{f^{2} \cos \theta} \\
& \simeq \frac{G^{\prime}}{G^{2}} \times \frac{2\left(m_{t \chi}^{2}+m_{\chi \chi}^{2}\right)}{f^{2}} \\
m_{h_{t}^{0}}^{2} & =\frac{2\left\langle\bar{\chi}_{R} \tilde{\chi}_{L}\right\rangle\left\langle\bar{\chi}_{R} \chi_{L}\right\rangle}{f^{2} \cos \theta} \times \sin ^{2} \theta \\
& =m_{A_{t}^{0}}^{2} \sin ^{2} \theta
\end{aligned}
$$

where the second equation of Eq.40 is obtained by expanding in terms of $G^{\prime} / G \ll 1$ and taking the leading nontrivial order. The mass of $h_{t}^{0}$ is proportional to $m_{t \chi}$ associated with the EWSB scale $v_{\mathrm{EW}}$ as in Eq. 29), just like the case of the SM Higgs boson, while the mass of $A_{t}^{0}$ is not. We may set the mass of $h_{t}^{0}$ to $\simeq 126 \mathrm{GeV}$;

$$
m_{h_{t}^{0}}=m_{A_{t}^{0}} \sin \theta \simeq 126 \mathrm{GeV} .
$$

Note also that $G^{\prime}>0$ assumed in Eq. (37) ensures the positiveness of squared masses for the Top-Mode Pseudos, $m_{h_{t}^{0}}^{2}>0$ and $m_{A_{t}^{0}}^{2}>0$. In Appendix. A. we present an alternative derivation of the pseudo NGBs masses and the heavy Higgs mass $m_{H_{t}^{0}}$ based on the approach used in [16].

As was done in Eq. 28), we may construct a nonlinear Lagrangian valid for scales below $m_{H_{t}^{0}}$ described by the five NGBs based on the coset space in Eq. 23 including the explicit breaking effect from the $G^{\prime}$ - and $\Delta_{\chi \chi}$-terms in Eq.(31). To this end, we introduce the spurion fields $\chi_{1}$ and $\chi_{2}$ to write the $\mathcal{O}\left(p^{2}\right)$ potential terms corresponding to Eq.(31):

$$
\Delta \mathcal{L}_{\mathrm{NL} \sigma \mathrm{M}}=f^{2} \operatorname{tr}\left[c_{1}\left(R^{T} U\right)^{\dagger} \chi_{1}\left(R^{T} U\right)+c_{2}\left(\chi_{2}^{\dagger}\left(R^{T} U\right)+\left(R^{T} U\right)^{\dagger} \chi_{2}\right)\right]
$$

where $\chi_{1}$ and $\chi_{2}$ transform under the $\mathcal{G}$-symmetry as

$$
\chi_{1} \rightarrow g_{3 L} \cdot \chi_{1} \cdot g_{3 \tilde{L}}^{\dagger}, \quad \chi_{2} \rightarrow g_{3 L} \cdot \chi_{2} \cdot g_{1 R}^{\dagger} .
$$

The $\mathcal{G}$-symmetry is explicitly broken when the spurion fields acquire the vacuum expectation values,

$$
\left\langle\chi_{1}\right\rangle=\left\langle\chi_{2}\right\rangle=\Sigma
$$

so that the $c_{1}$ and $c_{2}$ terms break the $\mathcal{G}$ down to $U(2)_{q_{L}} \times U(1)_{\chi_{L}} \times U(1)_{\chi_{R}}$ and $U(2)_{q_{L}} \times U(1)_{V=\chi_{R}+\chi_{L}}$, respectively, in the same way as the $G^{\prime}$ and $\Delta_{\chi \chi}$ terms in Eq.(31) do. Matching to the tHiggs mass formula in Eq.(41), we then find the coefficients $c_{1}$ and $c_{2}$ to be

$$
c_{1}=-\frac{1}{2} m_{A_{t}^{0}}^{2}, \quad c_{2}=\frac{1}{2} m_{A_{t}^{0}}^{2} \cos \theta .
$$

\section{Fermion masses and Yukawa couplings}

$$
\text { 1. top and } t^{\prime} \text {-quark }
$$

Let us consider the top quark mass based on the Lagrangian Eq. 30 . After the spontaneous symmetry breaking by the nontrivial solutions of the gap equations, Eqs. (32), (33) and (34), the mass terms of the top quark $t$ and its flavor partner $\chi$ look like

$$
\mathcal{L}_{\text {kin. }}+\mathcal{L}^{4 f}+\left.\mathcal{L}^{h}\right|_{\text {mass }}=-\left(\begin{array}{ll}
\bar{t}_{L} & \bar{\chi}_{L}
\end{array}\right)\left(\begin{array}{cc}
0 & m_{t \chi} \\
0 & m_{\chi \chi}
\end{array}\right)\left(\begin{array}{c}
t_{R} \\
\chi_{R}
\end{array}\right)+\text { h.c. }
$$

From this we find the fermion mass eigenvalues as

$$
m_{t^{\prime}}^{2}=m_{\chi \chi}^{2}+m_{t \chi}^{2} \quad, \quad m_{t}^{2}=0,
$$


where the mass eigenstates $t^{\prime}$ and $t$ are given by

$$
t_{L}^{\prime}=\tilde{\chi}_{L}=t_{L} \sin \theta+\chi_{L} \cos \theta \quad, \quad t_{L}=\tilde{t}_{L}=t_{L} \cos \theta-\chi_{L} \sin \theta .
$$

Thus the top quark does not "feel" the EWSB by $\sin \theta \neq 0\left(m_{t \chi} \neq 0\right)$ and is still massless. This is essentially due to the residual symmetry, $U(2)_{q_{R}} \times U(1)_{V=\tilde{\chi}_{L}+\chi_{R}}\left(U(2)_{q_{R}}: t_{R} \leftrightarrow b_{R}\right)$, which forbids the couplings between $t_{R}$ and $\chi_{L}$ in the Lagrangian Eq. (30), hence no mass term for $\bar{\chi}_{L} t_{R}$ in Eq. 46$]$.

To make the model more realistic, we introduce a four-fermion interaction term which breaks the residual symmetry so as to allow $t_{R}$ to couple to $\chi_{L}$ :

$$
\mathcal{L}_{\text {kin. }}+\mathcal{L}^{4 f}+\mathcal{L}^{h}+\mathcal{L}^{t}
$$

where

$$
\mathcal{L}^{t}=G^{\prime \prime}\left(\bar{\chi}_{L} \chi_{R}\right)\left(\bar{t}_{R} \chi_{L}\right)+\text { h.c. } .
$$

As was done in Eq. (37), we also treat the $G^{\prime \prime}$ coupling to be perturbative,

$$
0<\frac{G^{\prime \prime}}{G}<1
$$

so that the symmetry breaking pattern $\mathcal{G} / \mathcal{H}=\left[U(3)_{\tilde{\psi}_{L}} \times U(1)_{\chi_{R}}\right] /\left[U(2)_{\tilde{\psi}_{L}} \times U(1)_{V}\right]$ is not destroyed (the vacuum aligned to this manifold): $\left\langle\bar{t}_{R} \chi_{L}\right\rangle_{G^{\prime \prime}=0}=\left\langle\bar{t}_{R} t_{L}\right\rangle_{G^{\prime \prime}=0}=0$ : The Dashen formula Eq. (38) with the $G^{\prime \prime}$ term in Eq. (49) leads to

$$
\begin{aligned}
\left.m_{h_{t}^{0}}^{2}\right|_{G^{\prime \prime}} & =\frac{1}{f^{2}}\left\langle 0\left|\left[i Q^{5},\left[i Q^{5},-\mathcal{L}^{t}\right]\right]\right| 0\right\rangle \\
& =\frac{G^{\prime \prime}}{f^{2}}\left[\left(\cos ^{2} \theta-\sin ^{2} \theta\right)\left\langle\overline{\tilde{\chi}}_{L} t_{R}\right\rangle\left\langle\bar{t}_{R} \tilde{\chi}_{L}\right\rangle+2 \sin \theta \cos \theta\left\langle\overline{\tilde{\chi}}_{L} \chi_{R}\right\rangle\left\langle\bar{t}_{R} \tilde{t}_{L}\right\rangle\right] \\
& \simeq G^{\prime \prime}\left[\left\langle\bar{\chi}_{R} \chi_{L}\right\rangle_{G^{\prime \prime}=0}\left\langle\bar{t}_{R} \chi_{L}\right\rangle_{G^{\prime \prime}=0}-\left\langle\bar{\chi}_{R} t_{L}\right\rangle_{G^{\prime \prime}=0}\left\langle\bar{t}_{R} t_{L}\right\rangle_{G^{\prime \prime}=0}\right]+\mathcal{O}\left(\left(G^{\prime \prime}\right)^{2}\right) \\
& =0+\mathcal{O}\left(\left(G^{\prime \prime}\right)^{2}\right),
\end{aligned}
$$

similarly for the mass of the CP-odd Top-Mode Pseudo, $A_{t}^{0}$. Here we have granted the vacuum saturation valid at the leading order of $1 / N_{c}$. Thus the Top-Mode Pseudo's masses are stable against the leading order correction of the explicit-breaking $G^{\prime \prime}$ term as dictated by the Dashen formula Eq.(51). The next-to leading order in the $G^{\prime \prime}$ perturbation, i.e., $\left(G^{\prime \prime} / G\right)^{2}$ corrections (which are also of the leading order of $1 / N_{c}$ ), will affect the masses, as will be discussed later (see around Eq. 779 ) $)$.

After the spontaneous symmetry breaking by $\left\langle\bar{\chi}_{R} \tilde{\chi}_{L}\right\rangle \neq 0$, i.e. $m_{\tilde{\chi} \chi}^{2}=m_{t \chi}^{2}+m_{\chi \chi}^{2} \neq 0$, we thus have the fermion mass matrix,

$$
\mathcal{L}_{\text {kin. }}+\mathcal{L}^{4 f}+\mathcal{L}^{h}+\left.\mathcal{L}^{t}\right|_{\text {mass }}=-\left(\begin{array}{cc}
\bar{t}_{L} & \bar{\chi}_{L}
\end{array}\right)\left(\begin{array}{cc}
0 & m_{t \chi} \\
\mu_{\chi t} & m_{\chi \chi}
\end{array}\right)\left(\begin{array}{c}
t_{R} \\
\chi_{R}
\end{array}\right)+\text { h.c. },
$$

where $\mu_{\chi t}=-G^{\prime \prime}\left\langle\bar{\chi}_{R} \chi_{L}\right\rangle$ is a dynamical mass coming from $\mathcal{L}^{t}$ in Eq.448. Note that the fermion mass matrix is identical to that discussed in top-seesaw models [21, 22]. The top quark and $t^{\prime}$-quark masses are given as the eigenvalues of the mass matrix in Eq. 52 ,

$$
\begin{aligned}
& m_{t^{\prime}}^{2}=\frac{m_{t \chi}^{2}+m_{\chi \chi}^{2}+\mu_{\chi t}^{2}}{2}\left[1+\sqrt{1-\frac{4 m_{t \chi}^{2} \mu_{\chi t}^{2}}{\left(m_{t \chi}^{2}+m_{\chi \chi}^{2}+\mu_{\chi t}^{2}\right)^{2}}}\right], \\
& m_{t}^{2}=\frac{m_{t \chi}^{2}+m_{\chi \chi}^{2}+\mu_{\chi t}^{2}}{2}\left[1-\sqrt{1-\frac{4 m_{t \chi}^{2} \mu_{\chi t}^{2}}{\left(m_{t \chi}^{2}+m_{\chi \chi}^{2}+\mu_{\chi t}^{2}\right)^{2}}}\right] .
\end{aligned}
$$

Now the top quark mass becomes nonzero and is proportional to $m_{t \chi}$ which breaks the electroweak symmetry, similarly to the mass of $h_{t}^{0}$ in Eq.41, while the $t^{\prime}$-quark mass is not. The corresponding mass eigenstates $\left(t, t^{\prime}\right)_{m}^{T}$ are related to the gauge (current) eigenstates $(t, \chi)_{g}^{T}$ by the orthogonal rotation keeping $m_{t}, m_{t^{\prime}} \geq 0$ [23]:

$$
\left(\begin{array}{c}
t_{L} \\
t_{L}^{\prime}
\end{array}\right)_{m}=\left(\begin{array}{cc}
c_{L}^{t} & -s_{L}^{t} \\
s_{L}^{t} & c_{L}^{t}
\end{array}\right)\left(\begin{array}{c}
t_{L} \\
\chi_{L}
\end{array}\right)_{g}=O_{L} \cdot\left(\begin{array}{c}
t_{L} \\
\chi_{L}
\end{array}\right)_{g}, \quad\left(\begin{array}{c}
t_{R} \\
t_{R}^{\prime}
\end{array}\right)_{m}=\left(\begin{array}{cc}
-c_{R}^{t} & s_{R}^{t} \\
s_{R}^{t} & c_{R}^{t}
\end{array}\right)\left(\begin{array}{c}
t_{R} \\
\chi_{R}
\end{array}\right)_{g}=O_{R} \cdot\left(\begin{array}{c}
t_{R} \\
\chi_{R}
\end{array}\right)_{g},
$$


where $c_{L(R)}^{t} \equiv \cos \theta_{L(R)}^{t}$ and $s_{L(R)}^{t} \equiv \sin \theta_{L(R)}^{t}$ which are given up to $\mathcal{O}\left(\left(G^{\prime \prime} / G\right)^{2}\right)$ as

$$
\begin{aligned}
& c_{L}^{t}=\frac{1}{\sqrt{2}}\left[1+\frac{m_{\chi \chi}^{2}-m_{t \chi}^{2}+\mu_{\chi t}^{2}}{m_{t^{\prime}}^{2}-m_{t}^{2}}\right]^{1 / 2} \simeq \cos \theta\left[1+\left(\frac{G^{\prime \prime}}{G}\right)^{2} \cos ^{2} \theta \sin ^{2} \theta\right], \\
& s_{L}^{t}=\frac{1}{\sqrt{2}}\left[1-\frac{m_{\chi \chi}^{2}-m_{t \chi}^{2}+\mu_{\chi t}^{2}}{m_{t^{\prime}}^{2}-m_{t}^{2}}\right]^{1 / 2} \simeq \sin \theta\left[1-\left(\frac{G^{\prime \prime}}{G}\right)^{2} \cos ^{4} \theta\right], \\
& c_{R}^{t}=\frac{1}{\sqrt{2}}\left[1+\frac{m_{\chi \chi}^{2}+m_{t \chi}^{2}-\mu_{\chi t}^{2}}{m_{t^{\prime}}^{2}-m_{t}^{2}}\right]^{1 / 2} \simeq 1-\frac{1}{2}\left(\frac{G^{\prime \prime}}{G}\right)^{2} \cos ^{4} \theta, \\
& s_{R}^{t}=\frac{1}{\sqrt{2}}\left[1-\frac{m_{\chi \chi}^{2}+m_{t \chi}^{2}-\mu_{\chi t}^{2}}{m_{t^{\prime}}^{2}-m_{t}^{2}}\right]^{1 / 2} \simeq \frac{G^{\prime \prime}}{G} \cos ^{2} \theta .
\end{aligned}
$$

Including the effect of the explicit breaking $G^{\prime \prime}$ term, we may thus add the fermion sector to the nonlinear Lagrangian constructed from Eqs. 28) and (43),

$$
\mathcal{L}_{\text {yuk. }}^{t, t^{\prime}}=-\frac{f}{\sqrt{2}}\left[y \bar{\psi}_{L}\left(R^{T} U\right) \psi_{R}+y_{\chi t} \bar{\psi}_{L}\left(\chi_{1} R^{T} U \chi_{3}\right) \psi_{R}+\text { h.c. }\right],
$$

where $\psi_{R}=\left(t_{R}, b_{R}, \chi_{R}\right)^{T}$. The spurion fields $\chi_{1}$ and $\chi_{3}$ have been introduced in Eq. 60, which transform as

$$
\chi_{1} \rightarrow g_{3 L} \cdot \chi_{1} \cdot g_{3 L}^{\dagger}, \quad \chi_{3} \rightarrow g_{1 R} \cdot \chi_{3} \cdot g_{1 R}^{\dagger},
$$

so that the Lagrangian Eq. 60 is invariant under the $\mathcal{G}$-symmetry, $U(3)_{\tilde{\psi}_{L}} \times U(1)_{\chi_{R}}$, and $U(2)_{q_{R}}$ symmetry. These symmetries are explicitly broken by the vacuum expectation values of the spurion fields,

$$
\left\langle\chi_{1}\right\rangle=\Sigma, \quad\left\langle\chi_{3}\right\rangle=\lambda_{4}
$$

in which the $\left\langle\chi_{1}\right\rangle$ breaks the $U(3)_{\psi_{L}}$ symmetry down to $U(2)_{\psi_{L}} \times U(1)_{\chi_{L}}$ and the $\left\langle\chi_{3}\right\rangle$ does the $U(2)_{q_{R}} \times U(1)_{\chi_{R}}$ down to $U(1)_{\chi_{R}=t_{R}}$. We thus see that the first term in Eq. (60) corresponds to the $G$-four fermion term in Eq.(5), which is invariant under the $\mathcal{G}$-symmetry, $U(3)_{\tilde{\psi}_{L}} \times U(1)_{\chi_{R}}$ and $U(2)_{q_{R}}$ symmetry, while the second term does to the $G^{\prime \prime}$-four fermion term, which explicitly breaks the $\mathcal{G}$ and $U(2)_{q_{R}}$ down to $U(2)_{q_{L}} \times U(1)_{\chi_{L}}$ and $U(1)_{t_{R}=\chi_{R}}$. The Yukawa couplings $y$ and $y_{\chi t}$ can be fixed by matching to the fermion mass matrix in Eq. $(52)$ as

$$
y^{2}=\frac{2\left(m_{t \chi}^{2}+m_{\chi \chi}^{2}\right)}{f^{2}} \quad, \quad y_{\chi t}^{2}=y^{2}\left(\frac{G^{\prime \prime}}{G}\right)^{2}=\frac{2 \mu_{\chi t}^{2}}{f^{2} \cos ^{2} \theta} .
$$

\section{Fermions other than top and $t^{\prime}$-quark}

In order to give masses to SM fermions other than the top quark, inspired by [11, 12, we may add the following four-fermion interactions to the Lagrangian Eq. 48]:

$$
\mathcal{L}^{\text {others }}=\sum_{\alpha=1,2} G_{t u}^{\alpha}\left(\bar{q}_{L}^{i} \chi_{R}\right)\left(\bar{u}_{R}^{\alpha} q_{L}^{\alpha, i}\right)+\sum_{\alpha=1,2,3} G_{t d}^{\alpha}\left(\bar{q}_{L}^{i} \chi_{R}\right)\left(i \tau^{2}\right)^{i j}\left(\bar{q}_{L}^{\alpha, j} d_{R}^{\alpha}\right)+\sum_{\alpha=1,2,3} G_{t e}^{\alpha}\left(\bar{q}_{L}^{i} \chi_{R}\right)\left(i \tau^{2}\right)^{i j}\left(\bar{l}_{L}^{\alpha, j} e_{R}^{\alpha}\right)+\text { h.c },(64)
$$

which are SM gauge invariant, where $\alpha=1,2,3$ denotes the index for the fermion generation and $i, j=1,2$ for the weak isospin. With the nonzero condensate $\left\langle\bar{\chi}_{R} t_{L}\right\rangle \neq 0\left(m_{t \chi} \neq 0\right)$ breaking the electroweak gauge symmetry, these SM fermions acquire their masses as

$$
m_{u^{\alpha}}=-G_{t u}^{\alpha}\left\langle\bar{\chi}_{R} t_{L}\right\rangle \quad, \quad m_{d^{\alpha}}=-G_{t d}^{\alpha}\left\langle\bar{\chi}_{R} t_{L}\right\rangle \quad, \quad m_{e^{\alpha}}=-G_{t e}^{\alpha}\left\langle\bar{\chi}_{R} t_{L}\right\rangle .
$$

Since the NGBs arise from $\bar{\chi}_{R} \psi_{L}$ as

$$
\bar{\chi}_{R} q_{L}^{i} \sim\left\langle\bar{\chi}_{R} \tilde{\chi}_{L}\right\rangle\left[R^{T} U\right]_{3 i},
$$


one can read off the fermion couplings to $h_{t}^{0}{ }^{2}$ :

$$
\left.\mathcal{L}_{\text {yuk. }}^{\text {others }}\right|_{h_{t}^{0}}=-\cos \theta\left[\sum_{\alpha=1,2} \frac{m_{u^{\alpha}}}{v_{\mathrm{EW}}} h_{t}^{0} \bar{u}^{\alpha} u^{\alpha}+\sum_{\alpha=1,2,3} \frac{m_{d^{\alpha}}}{v_{\mathrm{EW}}} h_{t}^{0} \bar{d}^{\alpha} d^{\alpha}+\sum_{\alpha=1,2,3} \frac{m_{e^{\alpha}}}{v_{\mathrm{EW}}} h_{t}^{0} \bar{e}^{\alpha} e^{\alpha}\right] .
$$

A full set of the particle content in the present model with the SM charge assignment free from the gauge anomaly is listed in Table. II.

\begin{tabular}{|c||c|c|c|}
\hline field & $S U(3)_{c}$ & $S U(2)_{L}$ & $U(1)_{Y}$ \\
\hline \hline$q_{L}=\left(\begin{array}{c}t_{L} \\
b_{L}\end{array}\right)$ & 3 & 2 & $1 / 6$ \\
$t_{R}$ & 3 & 1 & $2 / 3$ \\
$b_{R}$ & 3 & 1 & $-1 / 3$ \\
\hline \hline$l_{L}=\left(\begin{array}{c}\nu_{\tau L} \\
\tau_{L}\end{array}\right)$ & 1 & 2 & $-1 / 2$ \\
$\tau_{R}$ & 1 & 1 & -1 \\
\hline \hline$\chi_{L}$ & 3 & 1 & $2 / 3$ \\
$\chi_{R}$ & 3 & 1 & $2 / 3$ \\
\hline
\end{tabular}

TABLE II: Full particle contents and the charge assignments under the SM gauge group. All the fermions are represented in terms of the electroweak gauge eigenbasis.

\section{D. tHiggs}

We here discuss the coupling property of the tHiggs $h_{t}^{0}$ and the stability of the mass against radiative corrections. From Eqs. 28), 43), 60) and (66), we find the $h_{t}^{0}$ couplings to the SM particles,

$$
\begin{aligned}
& \mathcal{L}_{\mathrm{NL} \sigma \mathrm{M}}+\Delta \mathcal{L}_{\mathrm{NL} \sigma \mathrm{M}}+\mathcal{L}_{\text {yuk. }}^{t, t^{\prime}}+\left.\mathcal{L}_{\text {yuk. }}^{\text {others }}\right|_{h_{t}^{0}}=g_{h V V} \frac{v_{\mathrm{EW}}}{2}\left(g^{2} h_{t}^{0} W_{\mu}^{+} W^{-\mu}+\frac{g^{2}+g^{\prime 2}}{2} h_{t}^{0} Z_{\mu} Z^{\mu}\right) \\
& -g_{h h h} \frac{3 m_{h_{t}^{0}}^{2}}{v_{\mathrm{EW}}} \frac{\left(h_{t}^{0}\right)^{3}}{3 !}-g_{h h h h} \frac{3 m_{h_{t}^{0}}^{2}}{v_{\mathrm{EW}}^{2}} \frac{\left(h_{t}^{0}\right)^{4}}{4 !} \\
& -g_{h t t} \frac{m_{t}}{v_{\mathrm{EW}}} h_{t}^{0} \bar{t} t-g_{h b b} \frac{m_{b}}{v_{\mathrm{EW}}} h_{t}^{0} \bar{b} b-g_{h \tau \tau} \frac{m_{\tau}}{v_{\mathrm{EW}}} h_{t}^{0} \bar{\tau} \tau \\
& +\cdots \text {, }
\end{aligned}
$$

where

$$
\begin{aligned}
& g_{h V V}=g_{h h h}=g_{h b b}=g_{h \tau \tau}=\cos \theta \\
& g_{h h h h}=1-\frac{7}{3} \sin ^{2} \theta \\
& g_{h t t}=\frac{v_{\mathrm{EW}}}{m_{t}} \frac{y}{\sqrt{2}}\left[\left(c_{L}^{t} \cos \theta+s_{L}^{t} \sin \theta\right) s_{R}^{t}-s_{L}^{t} c_{R}^{t} \sin \theta\left(\frac{G^{\prime \prime}}{G}\right)\right]=\frac{2 \cos ^{2} \theta-1}{\cos \theta}+\mathcal{O}\left(\left(\frac{G^{\prime \prime}}{G}\right)^{2}\right) .
\end{aligned}
$$

From these, we see that the $h_{t}^{0}$ couplings to the $W, Z$ bosons and to the SM fermions become the same as the SM Higgs ones when we take the limit $\cos \theta \rightarrow 1$, i.e.,

$$
g_{h V V}=g_{h h h}=g_{h h h h}=g_{h t t}=g_{h b b}=g_{h \tau \tau}=g^{\mathrm{SM}}(=1)
$$

2 Those Yukawa interactions would give quadratically divergent corrections to the $h_{t}^{0}$ mass, which are, however, small enough due to the small Yukawa coupling for the lighter fermions, to be negligible compared to the terms in Eq. 79 p arising from the Top-Mode Pseudos, $t$ and $t^{\prime}$-loops. 
when

$$
\sin \theta=\frac{m_{t \chi}}{\sqrt{m_{t \chi}^{2}+m_{\chi \chi}^{2}}}=\frac{v_{\mathrm{EW}}}{f} \rightarrow 0, \quad \text { by } \quad f \rightarrow \infty \quad \text { with } \quad v_{\mathrm{EW}}=246 \mathrm{GeV} \quad \text { fixed } .
$$

Actually, this limit turns out to be favored by the current experiments as will be discussed in Sec. III A.

From Eqs. 28, 243), 60 and (66), we can also evaluate the quadratic divergent corrections to the $h_{t}^{0}$ mass at the one-loop level. The one-loop corrections of the leading order of the explicit breaking parameters $G^{\prime} / G, G^{\prime \prime} / G$ and $\alpha_{\mathrm{em}}=e^{2} /(4 \pi)$ with $e$ being the electromagnetic coupling, can be evaluated at the one-loop level of the nonlinear sigma model constructed from terms in Eqs. 28, 43,60 and 66 . The correction of $\mathcal{O}\left(G^{\prime \prime} / G\right)$ from $t$ and $t^{\prime}$ loops exactly cancel and do not contribute to the tHiggs mass, which is consistent with the Dashen formula in Eq. (51). Hence the leading order corrections in the perturbation with respect to the explicit breaking couplings only come from terms of $\mathcal{O}\left(G^{\prime} / G\right)=\mathcal{O}\left(m_{h_{t}^{0}}^{2}\right)$ and $\mathcal{O}\left(\alpha_{\mathrm{em}}\right)$, in which the quadratic divergent contributions dominate. Of these leading corrections, the electroweak gauge terms are actually highly suppressed by $\alpha_{\text {em }}$ compared to the corrections of $\mathcal{O}\left(\left(G^{\prime} / G\right) \Lambda_{\chi}^{2} /(4 \pi)^{2}\right)$ which arises from the Top-Mode Pseudo's $\left(h_{t}^{0}\right.$ and $\left.A_{t}^{0}\right)$ self-interaction sector, where $\Lambda_{\chi} \sim m_{H_{t}^{0}}$ is the cutoff of the nonlinear sigma model. Thus we evaluate the leading order corrections in the perturbation of $G^{\prime} / G, G^{\prime \prime} / G$ and $\alpha_{\mathrm{em}}$ to the tHiggs mass:

$$
\left.m_{h_{t}^{0}}^{2}\right|_{1-\text { loop }} ^{\mathcal{O}\left(G^{\prime} / G, G^{\prime \prime} / G, \alpha_{\mathrm{em}}\right)}=m_{h_{t}^{0}}^{2}\left[1+\frac{\Lambda_{\chi}^{2}}{(4 \pi)^{2} v_{\mathrm{EW}}^{2}} \frac{23}{16}\right]+\mathcal{O}\left(\frac{\alpha_{\mathrm{em}} \Lambda_{\chi}^{2}}{4 \pi}\right) .
$$

The size of $G^{\prime} / G$ correction (the second term in the square bracket) could be $\mathcal{O}(1)$, when we took $\Lambda_{\chi} \sim m_{H_{t}^{0}} \sim 4 \pi v_{\text {Ew }}$, and hence is potentially a large correction to the tHiggs mass, which might need some fine tuning.

Actually, the top and $t^{\prime}$-loop corrections arising as the next to leading order of $\mathcal{O}\left(\left(G^{\prime \prime} / G\right)^{2}\right)$ will be more significant to give the sizable contribution to the tHiggs mass at the one-loop order since $G^{\prime \prime} / G$ is numerically not very small in order to realize the reality, though those terms are potentially suppressed in terms of the $\left(G^{\prime \prime} / G\right)$-perturbation. The concrete estimate of the size of corrections to the tHiggs mass from those terms will be addressed in Sec. IV.

One might naively suspect from Eqs. (72) that the presence of quadratic divergent corrections to the $h_{t}^{0}$ mass causes the fine-tuning problem just like the SM Higgs boson case. However, it is not the case because the $h_{t}^{0}$ is $n a t u r a l$ in accordance with the original argument in [29]: If one takes the massless Higgs boson limit $\left(m_{h_{t}^{0}} \rightarrow 0\right)$ corresponding to $G^{\prime} \rightarrow 0, G^{\prime \prime} \rightarrow 0$ (and $G_{t u}^{\alpha} \rightarrow 0, G_{t d}^{\alpha} \rightarrow 0, G_{t e}^{\alpha} \rightarrow 0$ ) and electroweak gauge interactions are turned off in Eqs.28, (43) and (60) (and Eq. 66 ) , then the global $\mathcal{G}$-symmetry is restored, meaning that the symmetry is enhanced. In this limit the quadratic divergences disappear as well. Thus the $h_{t}^{0}$ mass is protected by the $\mathcal{G}$-symmetry just like the QCD pseudo NGBs $(\pi, K \cdots)$. In contrast, as is well known, the SM Higgs sector is unnatural since even if one

takes the massless Higgs boson limit $\left(m_{h_{\mathrm{SM}}^{0}} \rightarrow 0\right)$ the symmetry of the SM is not enhanced. In this sense, the $h_{t}^{0}$ is a natural Higgs.

Before closing this section, it is also worth mentioning the difference between the present model and little Higgs models 30 33. They are similar in the sense that one of NGBs associated with the spontaneous global symmetry breaking $(\mathcal{G} / \mathcal{H})$ is identified as the Higgs boson, and the Higgs mass is generated through the explicit breaking effect. Therefore, the Higgs mass is under control in both models as explained in the previous paragraph. The crucial difference, though, is that, in the case of little Higgs models, the electroweak symmetry is embedded as a subgroup of $\mathcal{H}$, while in the case of the present model, it is outside of $\mathcal{H}$, namely, the electroweak symmetry is broken by the dynamics which breaks $\mathcal{G}$ down to $\mathcal{H}$.

\section{PHENOMENOLOGIES}

In this section, we discuss several constraints from existing experimental results and phenomenological implications of the model.

\section{A. Phenomenological constraints on Top-Mode Pseudos}

Examining Eqs. 67) and 68, we see that the couplings of the tHiggs $h_{t}^{0}$ to the $W$ and $Z$ bosons deviate from the SM Higgs ones by

$$
\kappa_{V} \equiv \frac{g_{h V V}}{g_{h V V}^{\mathrm{SM}}}=\cos \theta
$$


where $V=W$ and $Z$. The current LHC data give the constraint on $\kappa_{V}$ to be $\kappa_{V}>0.94$ at $95 \%$ C.L. for the $126 \mathrm{GeV}$ Higgs boson [34]. Therefore, we obtain the following constraint on the angle $\theta$ :

$$
\cos \theta>0.94, \quad \sin \theta<0.34 .
$$

As noted in Eq. (71), the tHiggs couplings to the SM particles coincide with the SM Higgs ones when $\theta \ll 1$ : The deviation in the ratio of the couplings to fermions to those of the $W$ and $Z$ bosons, $g_{h f f} / g_{h V V}$, can be expanded in powers of $\theta$ as

$$
\begin{aligned}
& \frac{g_{h f f}}{g_{h V V}}=1, \quad \text { for } \quad f=\tau, b, \\
& \frac{g_{h t t}}{g_{h V V}} \simeq 1-\frac{3}{2} \theta^{2} .
\end{aligned}
$$

This and the current bound on $\theta$ in Eq. (74) imply that the highly precise measurement (by about $5 \%$ accuracy) would be required to distinguish the coupling properties between the SM Higgs and the tHiggs. It might be possible to make it by the high luminosity LHC or ILC [35].

Here, it is also worth giving some comments on the difference between the present low-energy effective theory (Eqs. 28), 43 and (60) and the two-Higgs doublet model since the top-seesaw model is often described by using a two Higgs doublet model as the low-energy effective theory [23, 24]. One difference is in the low-energy mass spectrum: As seen in SecIIB the low-energy mass spectrum in the present model has no charged Higgs bosons which the two-Higgs doublet model posses. The other would be the tree-level mass relation among the neutral Higgs bosons $\left(A_{t}^{0}\right.$ and $\left.h_{t}^{0}\right)$ as in Eq.42, which is absent in the usual two-Higgs doublet model and therefore may distinguish two models.

\section{B. $S, T$ parameters and the constraint on $t^{\prime}$-quark mass}

Looking at the current bound on $\theta$ in Eq. (74), we see that the coupling property of the $t^{\prime}$-quark arises mainly from the $S U(2)_{L}$ singlet $\chi$-quark which carries exactly the same charge as that of the right-handed top quark. The mass of $t^{\prime}$-quark can therefore be constrained from the Peskin-Takeuchi $S, T$-parameters [36, 37] ${ }^{3}$.

By taking $m_{t^{\prime}} \gg m_{t} \gg m_{b}$, the one-loop contributions from the $t^{\prime}$-quark to the $S, T$-parameters are evaluated as 22 ]

$$
\begin{aligned}
& S=\frac{3}{2 \pi}\left(s_{L}^{t}\right)^{2}\left[-\frac{1}{9} \ln \frac{x_{t^{\prime}}}{x_{t}}-\left(c_{L}^{t}\right)^{2} F\left(x_{t}, x_{t^{\prime}}\right)\right], \\
& T=\frac{3}{16 \pi s_{W}^{2} c_{W}^{2}}\left(s_{L}^{t}\right)^{2}\left[\left(s_{L}^{t}\right)^{2} x_{t^{\prime}}-\left(1+\left(c_{L}^{t}\right)^{2}\right) x_{t}+\left(c_{L}^{t}\right)^{2} \frac{2 x_{t^{\prime}} x_{t}}{x_{t^{\prime}}-x_{t}} \ln \frac{x_{t^{\prime}}}{x_{t}}\right],
\end{aligned}
$$

where $s_{W} \equiv \sin \theta_{W}\left(c_{W}^{2} \equiv 1-s_{W}^{2}\right)$ is the weak mixing angle and $x_{a} \equiv m_{a}^{2} / m_{Z}^{2},\left(a=t, t^{\prime}\right)$. The function $F(x, y)$ is given by 38 ]

$$
F(x, y)=\frac{5\left(x^{2}+y^{2}\right)-22 x y}{9(x-y)^{2}}+\frac{3 x y(x+y)-x^{3}-y^{3}}{3(x-y)^{3}} \ln \frac{x}{y} .
$$

The $S, T$-parameters in Eqs. 75 and 76 are calculated as a function of the two parameters, $s_{L}^{t}\left(c_{L}^{t}\right)$ and $m_{t^{\prime}}$ once we fix $m_{Z}, s_{W}\left(c_{W}\right)$ and $m_{t}$ to be the experimental values [20. Expanding Eqs. (53) and (54) in powers of $G^{\prime \prime} / G=\mu_{\chi t} / m_{\chi \chi}<1$ we express the ratio $m_{t} / m_{t^{\prime}}$ to the next to leading order of $G^{\prime \prime} / G$ :

$$
\frac{m_{t}}{m_{t^{\prime}}} \simeq \sin \theta \cos \theta\left(\frac{G^{\prime \prime}}{G}\right)\left[1-\cos ^{4} \theta\left(\frac{G^{\prime \prime}}{G}\right)^{2}\right]=\frac{\mu_{\chi t}}{m_{\chi \chi}} \sin \theta \cos \theta\left[1-\cos ^{4} \theta\left(\frac{\mu_{\chi t}}{m_{\chi \chi}}\right)^{2}\right] .
$$

Using this and Eq. (57), we can rewrite $s_{L}^{t}\left(c_{L}^{t}\right)$ in terms of $\theta$ and $G^{\prime \prime} / G$ to evaluate the $S$ and $T$ as a function of $\theta$ and $G^{\prime \prime} / G$. In Fig. 1 (left panel), we thus plot the $S, T$-parameters versus $\cos \theta$ for several values of $G^{\prime \prime} / G$, together with

\footnotetext{
3 Another possible constraint would be $t^{\prime}$-quark contribution to the $Z b_{L} \bar{b}_{L}$ coupling, which, however, turns out to be much milder than that from the $S, T$ parameters. This is due to the fact that the present model does not include $b^{\prime}$-like particle usually arising in a class of top-seesaw models with so-called bottom-seesaw mechanism 23, 24].
} 
95\% C.L. allowed region (inside of the ellipsis) on the $(S, T)$-plane [39]. We have taken into account the constraints on $\cos \theta$ in Eq. (74) from the current LHC Higgs search. From the right panel of Fig. 1. we read off the allowed $t^{\prime}$-quark mass,

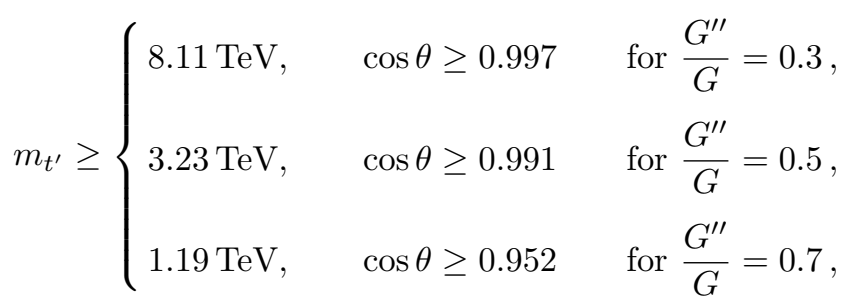

where the lower mass limits corresponds to the 95\% C.L. upper limit of the $S T$-ellipsis in the left panel of Fig. 1 . Note that the limit $\cos \theta \rightarrow 1(\sin \theta \rightarrow 0)$ corresponds to the decoupling limit of $t^{\prime}$-quark, $m_{t^{\prime}} \rightarrow \infty$ where $s_{L}^{t} \rightarrow 0$. The stringent phenomenological constraints on the $t^{\prime}$-quark thus come from the contribution to the $S, T$ parameters, which limit the mass to be $\gtrsim \mathcal{O}(\mathrm{TeV})$. Here $v_{\mathrm{EW}}=246 \mathrm{GeV}$ is realized when the cutoff scale of NJL dynamics is set as $\Lambda \simeq 66,223,480 \mathrm{TeV}$ for $G^{\prime \prime} / G=0.3,0.5,0.7$.
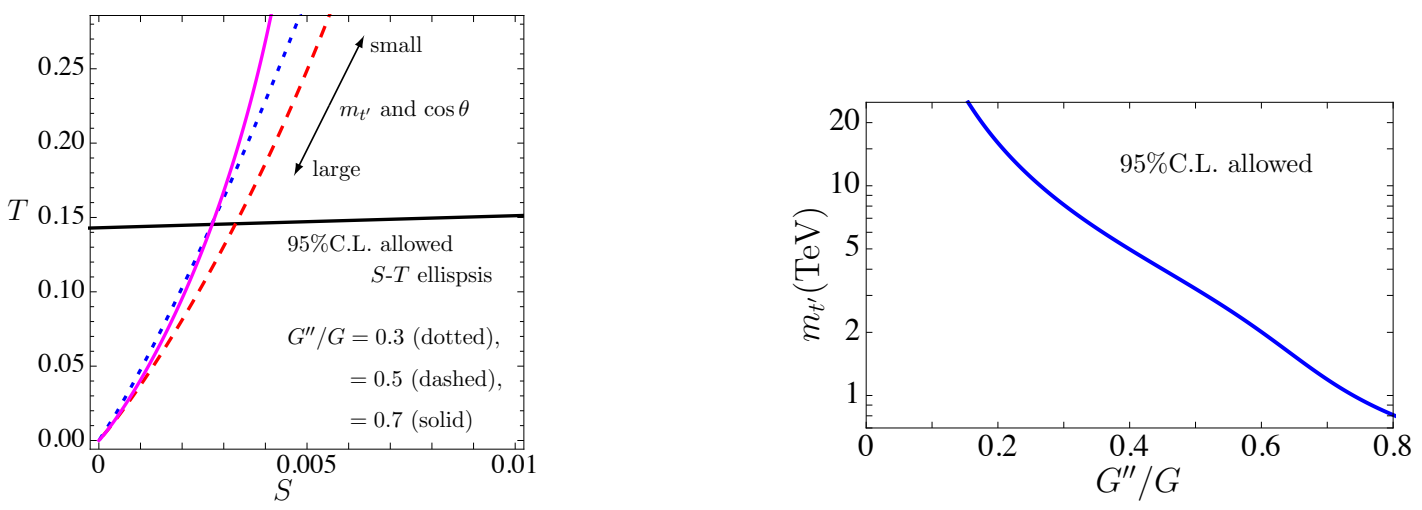

FIG. 1: Left panel: The $S, T$ constraints from Eqs. 75 and 76 on the $t^{\prime}$-quark mass in the $(S, T)$-plane for $G^{\prime \prime} / G=0.3$ (dotted), 0.5 (dashed), 0.7 (solid). The 95\% C.L. allowed region corresponds to an area lower than the solid curve (inside the $S-T$ ellipsis). The region of $S<0$ and $T<0$ are not displayed due to $c_{L}^{t} \leq 1^{a}$. Right panel: The $t^{\prime}$-quark mass versus $G^{\prime \prime} / G$ allowed by the $S, T$ constraints of the left panel. The $95 \%$ C.L. allowed region corresponds to an area upper than the blue curve.

\section{SUMMARY AND DISCUSSION}

In the spirit of the top quark condensation, we proposed a model which has a naturally light composite Higgs boson to be identified with the $126 \mathrm{GeV}$ Higgs discovered at the LHC. The tHiggs, a bound state of the top quark and its flavor (vector-like) partner, emerges as a pseudo Nambu-Goldstone boson (NGB), Top-Mode Pseudo, together with the exact NGBs to be absorbed into the $W$ and $Z$ bosons as well as another (heavier) Top-Mode Pseudo (CP-odd composite scalar, $A_{t}^{0}$ ). Those five composite (exact/pseudo) NGBs are dynamically produced simultaneously by a single supercritical four-fermion interaction having the $U(3)_{\tilde{\psi}_{L}} \times U(1)_{\chi_{R}}$ symmetry which includes the electroweak symmetry, where the vacuum is aligned by small explicit breaking term so as to break the symmetry down to a subgroup, $U(2)_{\tilde{\psi}_{L}} \times U(1)_{V=\chi_{L}+\chi_{R}}$, in a way not to retain the electroweak symmetry, in sharp contrast to the little Higgs models.

The $h_{t}^{0}$ couplings to the SM particles coincide with those of the SM Higgs boson in the limit $\sin \theta=v_{\mathrm{EW}} / f \rightarrow 0$ with $v_{\mathrm{EW}}$ being finite (Eqs.(67) and (71)). Even if the tHiggs coupling coincides with that of the SM Higgs, the virtue of our model is that the tHiggs $h_{t}^{0}$ is a bound state of the top quark and $\chi$-quark, and is natural in the sense that its mass is protected by the symmetry, in sharp contrast to the SM Higgs.

${ }^{a}$ We thank H. C. Cheng for pointing out the error omitting this obvious condition. 
One notable feature of our model is the prediction of the heavy CP-odd Higgs (without additional charged heavy Higgs in contrast to the two-doublet Higgs models). The mass of the $A_{t}^{0}$ is related to the tHiggs mass (Eq. (42)) at the tree-level of perturbations with respect to the explicit breaking effects, involving the size of deviation of couplings $(\sin \theta)$ to the electroweak gauge bosons from the SM Higgs ones. The CP-odd Top-Mode Pseudo $A_{t}^{0}$ does not couple to the $W$ and $Z$ bosons due to the CP-symmetry and the couplings to other SM particles are generically suppressed by $\sin \theta(<0.34)$. Hence the $A_{t}^{0}$ is distinguishable from that of the SM-like Higgs boson in the high-mass SM Higgs boson search at the LHC.

As noted around Eq. (72) the tHiggs would get the significant corrections of higher order in $G^{\prime \prime} / G$ to the mass from the top and $t^{\prime}$-quark as well as the Top-Mode pseudos' loops. In particular, the most sizable corrections would come from the top and $t^{\prime}$-loops only at sub-leading order $\mathcal{O}\left(\left(G^{\prime \prime} / G\right)^{2}\right)$ : Those one-loop corrections are dominated by the quadratic divergent terms as follows (for details of the computations, see Appendix. B):

$$
\begin{aligned}
\left.m_{h_{t}^{0}}^{2}\right|_{1-\text { loop }} ^{t, t^{\prime}} & =m_{h_{t}^{0}}^{2}+\frac{N_{c}}{(4 \pi)^{2}} y^{2}\left(\frac{G^{\prime \prime}}{G}\right)^{2}\left(2 \cos ^{2} \theta-1\right) \Lambda_{\chi}^{2} \\
& =m_{h_{t}^{0}}^{2}+\frac{N_{c}}{8 \pi^{2}}\left(\frac{\sqrt{2} m_{t}}{v_{\mathrm{EW}}}\right)^{2}\left(\frac{2 \cos ^{2} \theta-1}{\cos ^{2} \theta}\right) \Lambda_{\chi}^{2}\left[1+\mathcal{O}\left(\left(\frac{G^{\prime \prime}}{G}\right)^{2}\right)\right],
\end{aligned}
$$

where the first line is an exact result without further higher order corrections in $G^{\prime \prime} / G$ and in the second line we used $m_{t}^{2}=\left(y^{2} f^{2} / 2\right) \sin ^{2} \theta \cos ^{2} \theta\left(G^{\prime \prime} / G\right)^{2}\left[1+\mathcal{O}\left(\left(G^{\prime \prime} / G\right)^{2}\right)\right]$ which can be derived from Eq. 54 with Eq. 63) and $v_{\mathrm{EW}}=f \sin \theta$, and the chiral symmetry breaking scale $\Lambda_{\chi} \sim m_{H_{t}^{0}}$ is the cutoff of the nonlinear sigma model constructed from Eqs. 28), (43), (60) and (66). From Eq. (79), we see that the perturbative $G^{\prime \prime} / G$ corrections contribute to the tHiggs mass at the order of $\left.\mathcal{O}\left(G^{\prime} / G\right)^{2}\right)$, in accord with the Dashen formula Eq. (51). Note the amazing cancellation of the quadratic divergent terms among the top and $t^{\prime}$-quark loops when the mixing angle $\theta$ reaches an ideal amount, $\cos \theta=1 / \sqrt{2}$. However, to be consistent with the current Higgs coupling measurement at the LHC, the $\theta$ is actually strongly constrained to be $\cos \theta>0.94$ (see Eq. 74 ), which is somewhat far from $\cos \theta=1 / \sqrt{2} \simeq 0.71$. Thus the ideal mixing cannot reproduce the reality.

One possibility to make the present model realistic would be to pull the $t^{\prime}$-quark mass down to a low scale in such a way that the $t^{\prime}$-quark can be integrated out. In that case, we may take the $t^{\prime}$-quark mass to be the cutoff of the nonlinear sigma model, $\Lambda_{\chi}$, say $\Lambda_{\chi}=m_{t^{\prime}} \simeq 1.2 \mathrm{TeV}$ which is consistent with the $S, T$-parameter constraints in Eq. (78) for $\cos \theta=0.952$. Then the one-loop quadratic divergent corrections only come from the tHiggs and $A_{t}^{0}$ loops as in Eq. (72) and top loops involving some effective $h_{t}^{0}-h_{t}^{0}-t-t$ vertices induced from integrating out the $t^{\prime}$-quark (see Appendix. B). Thus we find the mass shift (with $\Lambda_{\chi}$ replaced by $m_{t^{\prime}}$ ),

$$
\left.m_{h_{t}^{0}}^{2}\right|_{1-\text { loop }} ^{t, h_{t}^{0}, A_{t}^{0}}=m_{h_{t}^{0}}^{2}\left[1+\frac{m_{t^{\prime}}^{2}}{(4 \pi)^{2} v_{\mathrm{EW}}^{2}} \frac{23}{16}\right]-\frac{3}{8 \pi^{2}}\left(\frac{\sqrt{2} m_{t}}{v_{\mathrm{EW}}}\right)^{2} \frac{1-6 \cos ^{2} \theta+6 \cos ^{4} \theta}{\cos ^{2} \theta} m_{t^{\prime}}^{2}\left[1+\mathcal{O}\left(\left(\frac{G^{\prime \prime}}{G}\right)^{2}\right)\right] .
$$

Note the negative correction from the top quark for $\cos \theta=0.952$, in the absence of the $t^{\prime}$-quark loop contributions. Note also that the result differs from that coming only from the top loop, since the $t^{\prime}$-quark effects are not totally decoupled via equation of motion as manifested in the induced vertex which never exists in the theory having no $t^{\prime}$-quark from the onset. We thus achieve the desired tHiggs mass around $\simeq 126 \mathrm{GeV}$ at the one-loop level, when the cutoff $\Lambda_{\chi}=m_{t^{\prime}}$ is set to $\simeq 1.2 \mathrm{TeV}$ in which case we have the tree-level mass $\left.m_{h_{t}^{0}}\right|_{\text {tree }} \simeq 200 \mathrm{GeV}$.

As seen from the explicit one-loop computation for the $A_{t}^{0}$ mass given in Appendix. B. the mixing angle $\cos \theta=0.952$ is large enough to highly suppress the one-loop corrections to the $A_{t}^{0}$ mass, so that we may take $\left.\left.m_{A_{t}^{0}}\right|_{\text {tree }} \simeq m_{A_{t}^{0}}\right|_{1-\text { loop }} ^{t, h_{t}^{0}, A_{t}^{0}}$ from Eq. 80 . Using the tree-level mass relation among $h_{t}^{0}$ and $A_{t}^{0}$ together with the tree-level tHiggs mass $\simeq 200 \mathrm{GeV}$, we then find

$$
\left.\left.m_{A_{t}^{0}}\right|_{\text {tree }} \simeq m_{A_{t}^{0}}\right|_{1-\text { loop }} ^{t, h_{t}^{0}, A_{t}^{0}} \simeq 700 \mathrm{GeV} .
$$

The scenario in the above would be phenomenologically interesting, where the Top-Mode Pseudos $\left(h_{t}^{0}, A_{t}^{0}\right)$ (and heavy top Higgs $H_{0}^{t}$ with the mass $\simeq 2 m_{t^{\prime}} \simeq 2.4 \mathrm{TeV}$ ) as well as the $t^{\prime}$-quark have masses accessible at the LHC. Actually, the direct searches for $t^{\prime}$-quark at the LHC [0 43, have placed the limit, $m_{t^{\prime}} \geq 1 \mathrm{TeV}$, which is available also to the $t^{\prime}$-quark in the present model. However, in addition to usual $t^{\prime}$-quark searches as reported in [40 43, a decay channel $t^{\prime} \rightarrow t A_{t}^{0}$ would be a characteristic signature of the $t^{\prime}$-quark in the present model. More on the detailed phenomenological study is to be pursued in the future.

To summarize, the present model predicts the following five masses:

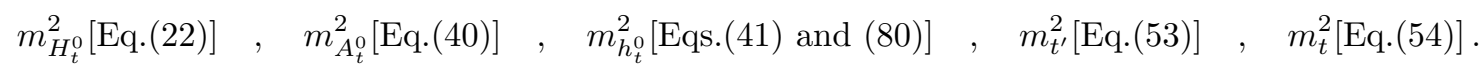


These masses are controlled by the five model parameters:

$$
G, G^{\prime}, \Delta_{\chi \chi}, G^{\prime \prime}, \Lambda .
$$

By tuning these model parameters, we can thus realize the mass hierarchies:

$$
\Lambda^{2}>m_{H_{t}^{0}}^{2} \sim m_{t^{\prime}}^{2} \gg m_{A_{t}^{0}}^{2}>m_{h_{t}^{0}}^{2} \sim m_{t}^{2} .
$$

The hierarchy $m_{H_{t}^{0}}^{2} \gg m_{A_{t}^{0}}^{2}$ is realized by tuning $(0<) G^{\prime} / G \ll 1$ as in Eq. 40 . The CP-even Top-Mode Pseudo (tHiggs) mass $m_{h_{t}^{0}}$ is smaller than the CP-odd Top-Mode Pseudo mass $m_{A_{t}^{0}}$ due to the mass relation in Eq. 41.). The fermion mass hierarchy $m_{t^{\prime}}>m_{t}$ is realized by taking $(0<) G^{\prime \prime} / G<1$ (see Eqs. (53) and (54)).

When we set $\Lambda=480 \mathrm{TeV}$ and take $G / G_{\text {crit }}-1 \simeq 10^{-4}$ to get $m_{t^{\prime}} \simeq 1.2 \mathrm{TeV}, m_{H_{t}^{0}} \simeq 2 m_{t^{\prime}} \simeq 2.4 \mathrm{TeV}$. Taking $v_{\mathrm{EW}}=246 \mathrm{GeV}$, which determines $m_{t \chi}$ through Eq. 299, and setting $G^{\prime} / G \simeq 10^{-5}$ and $G^{\prime \prime} / G \simeq 0.7$, we have $m_{t} \simeq 173 \mathrm{GeV}$ and $\left.m_{h_{t}^{0}}^{t}\right|_{1-\text { loop }} ^{t} \simeq 126 \mathrm{GeV}$ where $\left.m_{h_{t}^{0}}\right|_{\text {tree }} \simeq 200 \mathrm{GeV}$. Thus the phenomenologically favored situation as above can be realized from the original four-fermion dynamics.

\section{Acknowledments}

We would like to thank Hiroshi Ohki for useful discussions. This work was supported by the JSPS Grant-in-Aid for Scientific Research (S) \#22224003 and (C) \#23540300 (K.Y.).

\section{Appendix A: Alternative derivation of Top-Mode Pseudo mass formulas}

In this appendix we derive the mass formulas for the Top-Mode Pseudos in Eqs. 400 and (41) based on the BardeenHill-Lindner approach [16].

Introducing the auxiliary fields $\phi_{t \chi} \sim \bar{\chi}_{R} t_{L}, \phi_{b \chi} \sim \bar{\chi}_{R} b_{L}$ and $\phi_{\chi \chi} \sim \bar{\chi}_{R} \chi_{L}$, we rewrite the Lagrangian Eq. (30) into a linear sigma model-like form including the $1 / N_{c}$-leading corrections renormalized at the scale $\mu(<\Lambda)$,

$$
\mathcal{L}_{\mathrm{BHL}}=\mathcal{L}_{\text {kin. }}-\frac{1}{\sqrt{Z}}\left[\left(\begin{array}{ll}
\bar{t}_{L} & \bar{b}_{L}
\end{array}\right)\left(\begin{array}{c}
\phi_{t \chi} \\
\phi_{b \chi}
\end{array}\right) \chi_{R}+\bar{\chi}_{L} \phi_{\chi \chi} \chi_{R}+\text { h.c. }\right]+\mathcal{L}_{\mathrm{L} \sigma \mathrm{M}},
$$

where

$$
\begin{aligned}
\mathcal{L}_{\mathrm{L} \sigma \mathrm{M}} & =\left|D_{\mu}\left(\begin{array}{c}
\phi_{t \chi} \\
\phi_{b \chi}
\end{array}\right)\right|^{2}+\left|\partial_{\mu} \phi_{\chi \chi}\right|^{2}-V(\phi), \\
D_{\mu}\left(\begin{array}{c}
\phi_{t \chi} \\
\phi_{b \chi}
\end{array}\right) & =\left(\partial_{\mu}-i g W_{\mu}^{a} \frac{\sigma^{a}}{2}+i g^{\prime} \frac{1}{2} B_{\mu}\right)\left(\begin{array}{c}
\phi_{t \chi} \\
\phi_{b \chi}
\end{array}\right),
\end{aligned}
$$

and

$$
\begin{gathered}
V(\phi)=M^{2}\left[\phi_{t \chi}^{\dagger} \phi_{t \chi}+\phi_{b \chi}^{\dagger} \phi_{b \chi}+\phi_{\chi \chi}^{\dagger} \phi_{\chi \chi}\right]+\lambda\left[\phi_{t \chi}^{\dagger} \phi_{t \chi}+\phi_{b \chi}^{\dagger} \phi_{b \chi}+\phi_{\chi \chi}^{\dagger} \phi_{\chi \chi}\right]^{2} \\
+\Delta M^{2} \phi_{\chi \chi}^{\dagger} \phi_{\chi \chi}-C_{\chi \chi}\left[\phi_{\chi \chi}^{\dagger}+\phi_{\chi \chi}\right] \\
Z=\frac{1}{\lambda}=\frac{N_{c}}{16 \pi^{2}} \ln \frac{\Lambda^{2}}{\mu^{2}}, \quad M^{2}=\frac{1}{Z}\left(\frac{1}{G}-\frac{N_{c}}{8 \pi^{2}} \Lambda^{2}\right) \\
\Delta M^{2}=\frac{1}{Z}\left(\frac{1}{G-G^{\prime}}-\frac{1}{G}\right), \quad C_{\chi \chi}=\frac{1}{\sqrt{Z}} \frac{\Delta_{\chi \chi}}{G-G^{\prime}}
\end{gathered}
$$

We define the vacuum expectation values corresponding to Eq. (9),

$$
\left\langle\phi_{t \chi}\right\rangle=\frac{f \sin \theta}{\sqrt{2}} \equiv \frac{v_{t \chi}}{\sqrt{2}} \quad, \quad\left\langle\phi_{b \chi}\right\rangle=0 \quad, \quad\left\langle\phi_{\chi \chi}\right\rangle=\frac{f \cos \theta}{\sqrt{2}} \equiv \frac{v_{\chi \chi}}{\sqrt{2}},
$$


and the dynamical masses,

$$
m_{A B}=\frac{1}{\sqrt{Z}}\left\langle\phi_{A B}\right\rangle \quad \text { for } \quad A, B=t, b, \chi
$$

The stationary conditions, corresponding to the gap equations Eqs. 32$]$ and $[33]$, are obtained from the potential $V(\phi)$ to be

$$
\begin{aligned}
\frac{\partial V}{\partial v_{t \chi}}=0 \quad \Leftrightarrow \quad m_{t \chi}=m_{t \chi} \frac{N_{c} G}{8 \pi^{2}}\left[\Lambda^{2}-\left(m_{t \chi}^{2}+m_{\chi \chi}^{2}\right) \ln \frac{\Lambda^{2}}{m_{t \chi}^{2}+m_{\chi \chi}^{2}}\right], \\
\frac{\partial V}{\partial v_{\chi \chi}}=0 \quad \Leftrightarrow \quad m_{\chi \chi}=\Delta_{\chi \chi}+m_{\chi \chi} \frac{N_{c}\left(G-G^{\prime}\right)}{8 \pi^{2}}\left[\Lambda^{2}-\left(m_{t \chi}^{2}+m_{\chi \chi}^{2}\right) \ln \frac{\Lambda^{2}}{m_{t \chi}^{2}+m_{\chi \chi}^{2}}\right] .
\end{aligned}
$$

We next parametrize the neutral scalar fields $\phi_{t \chi}$ and $\phi_{\chi \chi}$ as

$$
\phi_{t \chi}=\frac{v_{t \chi}+\operatorname{Re} \phi_{t \chi}+i \operatorname{Im} \phi_{t \chi}}{\sqrt{2}} \quad, \quad \phi_{\chi \chi}=\frac{v_{\chi \chi}+\operatorname{Re} \phi_{\chi \chi}+i \operatorname{Im} \phi_{\chi \chi}}{\sqrt{2}} .
$$

Taking into account the stationary conditions Eqs. A6 and (A7), we find the mass terms of $\left(\operatorname{Re} \phi_{t \chi}, \operatorname{Re} \phi_{\chi \chi}, \operatorname{Im} \phi_{\chi \chi}\right)$ in the effective potential Eq. A3],

$$
-\frac{1}{2} m_{A_{t}^{0}}^{2}\left(\operatorname{Im} \phi_{\chi \chi}\right)^{2}-\frac{1}{2}\left(\begin{array}{ll}
\operatorname{Re} \phi_{t \chi} & \operatorname{Re} \phi_{\chi \chi}
\end{array}\right)\left(\begin{array}{cc}
4 m_{t \chi}^{2} & 4 m_{t \chi} m_{\chi \chi} \\
4 m_{t \chi} m_{\chi \chi} & 4 m_{\chi \chi}^{2}+m_{A_{t}^{0}}^{2}
\end{array}\right)\left(\begin{array}{c}
\operatorname{Re} \phi_{t \chi} \\
\operatorname{Re} \phi_{\chi \chi}
\end{array}\right)
$$

where $\operatorname{Im} \phi_{\chi \chi} \equiv A_{t}^{0}$ and the $A_{t}^{0}$ mass $m_{A_{t}^{0}}$ is given by expanding terms in powers of $G^{\prime} / G \ll 1$ as

$$
\begin{aligned}
m_{A_{t}^{0}}^{2} & =\Delta M^{2} \\
& =\left(\frac{1}{G-G^{\prime}}-\frac{1}{G}\right) \times \frac{16 \pi^{2}}{N_{c} \ln \left(\Lambda^{2} /\left(m_{t \chi}^{2}+m_{\chi \chi}^{2}\right)\right)} \\
& \simeq \frac{16 \pi^{2}}{G N_{c} \ln \left(\Lambda^{2} /\left(m_{t \chi}^{2}+m_{\chi \chi}^{2}\right)\right)}\left(\frac{G^{\prime}}{G}\right)\left[1+\mathcal{O}\left(\left(\frac{G^{\prime}}{G}\right)^{2}\right)\right] \\
& \simeq \frac{2\left(m_{t \chi}^{2}+m_{\chi \chi}^{2}\right)}{G f^{2}}\left(\frac{G^{\prime}}{G}\right)\left[1+\mathcal{O}\left(\left(\frac{G^{\prime}}{G}\right)^{2}\right)\right]
\end{aligned}
$$

where the renormalization scale $\mu$ has been set to $\left(m_{t \chi}^{2}+m_{\chi \chi}^{2}\right)^{1 / 2}$ and use has been made of the Pagels-Stokar formula for the decay constant $f$ given in Eq. 21 . For $G^{\prime} / G \ll 1$ we may take $m_{A_{t}^{0}} \ll m_{\tilde{\chi} \chi}=\left(m_{t \chi}^{2}+m_{\chi \chi}^{2}\right)^{1 / 2}$ so that the scalar mass in the last term of Eq. A9 can be diagonalized up to terms of $\mathcal{O}\left(m_{A_{t}^{0}}^{4} / m_{\tilde{\chi} \chi}^{2}\right)$ as

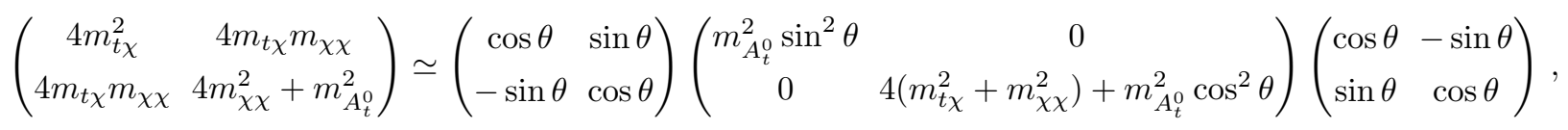

where $\tan \theta \equiv m_{t \chi} / m_{\chi \chi}$ and the corresponding mass eigenstates $h_{t}^{0}$ and $H_{t}^{0}$ with $m_{H_{t}^{0}}>m_{h_{t}^{0}}$ are given as

$$
\left(\begin{array}{c}
h_{t}^{0} \\
H_{t}^{0}
\end{array}\right)=\left(\begin{array}{cc}
\cos \theta & -\sin \theta \\
\sin \theta & \cos \theta
\end{array}\right)\left(\begin{array}{c}
\operatorname{Re} \phi_{t \chi} \\
\operatorname{Re} \phi_{\chi \chi}
\end{array}\right) .
$$

Thus we find the masses of the two CP-even neutral scalar mesons up to terms of $\mathcal{O}\left(m_{A_{t}^{0}}^{4} / m_{\tilde{\chi} \chi}^{2}\right)$ :

$$
m_{h_{t}^{0}}^{2} \simeq m_{A_{t}^{0}}^{2} \sin ^{2} \theta \quad, \quad m_{H_{t}^{0}}^{2} \simeq 4\left(m_{t \chi}^{2}+m_{\chi \chi}^{2}\right) \text {. }
$$

Eqs. A10 and A11 exactly reproduce the Top-Mode Pseudo mass formulas in Eqs.40 and 41 obtained from the nonlinear Lagrangian with the heavy top-Higgs integrated out. 


\section{Appendix B: $t, t^{\prime}$-loop corrections to the Top-Mode Pseudo masses}

In this appendix, we shall compute the quadratic divergent corrections to the Top Mode Pseudos $\left(h_{t}^{0}, A_{t}^{0}\right)$ arising from the top and $t^{\prime}$-loops as the $1 / N_{c}$-leading contribution in Eqs. 79 ) and 80 .

We start with the Yukawa sector in Eq.60,

$$
\mathcal{L}_{\text {yuk. }}^{t, t^{\prime}}=-\frac{f}{\sqrt{2}}\left[y \bar{\psi}_{L}\left(R^{T} U\right) \psi_{R}+y_{\chi} \bar{\psi}_{L}\left(\chi_{1} R^{T} U \chi_{3}\right) \psi_{R}+\text { h.c. }\right] .
$$

From this Lagrangian, we find the couplings relevant to the one-loop corrections in the basis of the mass-eigenstates $\left(t, t^{\prime}\right)_{m}$ :

$$
\begin{aligned}
\left.\mathcal{L}_{\text {yuk. }}^{t, t^{\prime}}\right|_{h_{t}^{0}}= & -y_{h t t} h_{t}^{0} \bar{t} t-y_{h t^{\prime} t^{\prime}} h_{t}^{0} \bar{t}^{\prime} t^{\prime}-g_{h t_{L} t_{R}^{\prime}} h_{t}^{0}\left(\bar{t}_{L} t_{R}^{\prime}+\text { h.c. }\right)-g_{h t_{R} t_{L}^{\prime}} h_{t}^{0}\left(\bar{t}_{R} t_{L}^{\prime}+\text { h.c. }\right) \\
& -g_{h h t t} h_{t}^{0} h_{t}^{0} \bar{t} t-g_{h h t^{\prime} t^{\prime}} h_{t}^{0} h_{t}^{0} \bar{t}^{\prime} t^{\prime}+\cdots
\end{aligned}
$$

and

$$
\begin{aligned}
\left.\mathcal{L}_{\text {yuk. }}^{t, t^{\prime}}\right|_{A_{t}^{0}}= & -y_{A t t} A_{t}^{0} \bar{t} \gamma_{5} t-y_{A t^{\prime} t^{\prime}} A_{t}^{0} \bar{t}^{\prime} \gamma_{5} t^{\prime}-g_{A t_{L} t_{R}^{\prime}} A_{t}^{0} \bar{t} \gamma_{5} t^{\prime}-g_{A t_{R} t_{L}^{\prime}} A_{t}^{0} \bar{t} \gamma_{5} t^{\prime} \\
& -g_{A A t t} A_{t}^{0} A_{t}^{0} \bar{t} \gamma_{5} t-g_{A A t^{\prime} t^{\prime}} A_{t}^{0} A_{t}^{0} \bar{t}^{\prime} \gamma_{5} t^{\prime}+\cdots
\end{aligned}
$$

where

$$
\begin{aligned}
y_{h t t} & =\frac{y}{\sqrt{2}}\left[\left(c_{L}^{t} \cos \theta+s_{L}^{t} \sin \theta\right) s_{R}^{t}-s_{L}^{t} c_{R}^{t} \sin \theta\left(\frac{G^{\prime \prime}}{G}\right)\right], \\
y_{h t^{\prime} t^{\prime}} & =\frac{y}{\sqrt{2}}\left[\left(s_{L}^{t} \cos \theta-c_{L}^{t} \sin \theta\right) c_{R}^{t}-c_{L}^{t} s_{R}^{t} \sin \theta\left(\frac{G^{\prime \prime}}{G}\right)\right], \\
g_{h t_{L} t_{R}^{\prime}} & =\frac{y}{\sqrt{2}}\left[\left(c_{L}^{t} \cos \theta+s_{L}^{t} \sin \theta\right) c_{R}^{t}+s_{L}^{t} s_{R}^{t} \sin \theta\left(\frac{G^{\prime \prime}}{G}\right)\right], \\
g_{h t_{L}^{\prime} t_{R}} & =\frac{y}{\sqrt{2}}\left[\left(s_{L}^{t} \cos \theta-c_{L}^{t} \sin \theta\right) s_{R}^{t}+c_{L}^{t} c_{R}^{t} \sin \theta\left(\frac{G^{\prime \prime}}{G}\right)\right], \\
g_{h h t t} & =\frac{y}{2 \sqrt{2} f}\left[\left(c_{L}^{t} \sin \theta-s_{L}^{t} \cos \theta\right) s_{R}^{t}+s_{L}^{t} c_{R}^{t} \cos \theta\left(\frac{G^{\prime \prime}}{G}\right)\right], \\
g_{h h t^{\prime} t^{\prime}} & =\frac{y}{2 \sqrt{2} f}\left[\left(s_{L}^{t} \sin \theta+c_{L}^{t} \cos \theta\right) c_{R}^{t}+c_{L}^{t} s_{R}^{t} \cos \theta\left(\frac{G^{\prime \prime}}{G}\right)\right],
\end{aligned}
$$

and

$$
\begin{aligned}
y_{A t t} & =\frac{i y}{\sqrt{2}}\left[-s_{L}^{t} s_{R}^{t}+s_{L}^{t} c_{R}^{t}\left(\frac{G^{\prime \prime}}{G}\right)\right], \\
y_{A t^{\prime} t^{\prime}} & =\frac{i y}{\sqrt{2}}\left[c_{L}^{t} c_{R}^{t}+c_{L}^{t} s_{R}^{t}\left(\frac{G^{\prime \prime}}{G}\right)\right], \\
g_{A t_{L} t_{R}^{\prime}} & =\frac{i y}{\sqrt{2}}\left[-s_{L}^{t} c_{R}^{t}-s_{L}^{t} s_{R}^{t}\left(\frac{G^{\prime \prime}}{G}\right)\right], \\
g_{A t_{L}^{\prime} t_{R}} & =\frac{i y}{\sqrt{2}}\left[c_{L}^{t} s_{R}^{t}-c_{L}^{t} c_{R}^{t}\left(\frac{G^{\prime \prime}}{G}\right)\right], \\
g_{A A t t} & =g_{h h t t}+\frac{3 y}{4 \sqrt{2} f} \sin \theta \cos \theta\left[-\left(c_{L}^{t} \cos \theta+s_{L}^{t} \sin \theta\right) s_{R}^{t}+s_{L}^{t} c_{R}^{t} \sin \theta\left(\frac{G^{\prime \prime}}{G}\right)\right], \\
g_{A A t^{\prime} t^{\prime}} & =g_{h h t^{\prime} t^{\prime}}+\frac{3 y}{4 \sqrt{2} f} \sin \theta \cos \theta\left[-\left(s_{L}^{t} \cos \theta-c_{L}^{t} \sin \theta\right) c_{R}^{t}+c_{L}^{t} s_{R}^{t} \sin \theta\left(\frac{G^{\prime \prime}}{G}\right)\right] .
\end{aligned}
$$

The one-loop corrections arise from Feynman graphs involving the top and $t^{\prime}$-loops as depicted in Fig. 2 The quadratic divergent corrections to the Top-Mode Pseudo masses are thus calculated to be

$$
\delta m_{h_{t}^{0}, A_{t}^{0}}^{2}=\frac{N_{c}}{8 \pi^{2}} \Lambda_{\chi}^{2} \cdot \mathcal{C}_{h, A}
$$



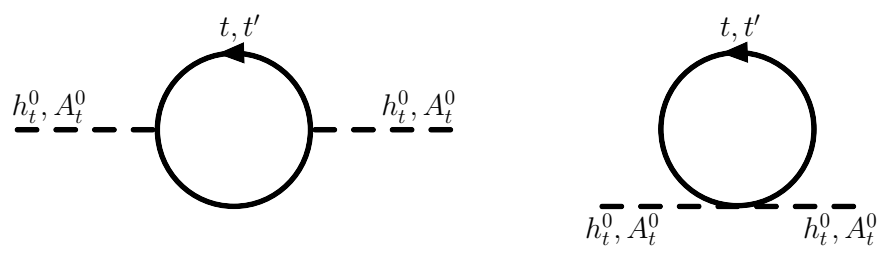

FIG. 2: The one-loop diagrams contributing to $h_{t}^{0}, A_{t}^{0}$ masses as the quadratic divergent corrections up to $\mathcal{O}\left(\left(G^{\prime \prime} / G\right)^{2}\right)$.

where the chiral symmetry breaking scale $\Lambda_{\chi}$ is the cutoff of the nonlinear sigma model constructed from Eqs. 28, (43), 60) and (66) and

$$
\begin{aligned}
\mathcal{C}_{h} & =-y_{h t t}^{2}-y_{h t^{\prime} t^{\prime}}^{2}-g_{h t_{L} t_{R}^{\prime}}^{2}-g_{h t_{L}^{\prime} t_{R}}^{2}+g_{h h t t}^{2}+g_{h h t^{\prime} t^{\prime}}^{2} \\
& =\frac{y^{2}}{2}\left(\frac{G^{\prime \prime}}{G}\right)^{2}\left(2 \cos ^{2} \theta-1\right), \\
\mathcal{C}_{A} & =-y_{A t t}^{2}-y_{A t^{\prime} t^{\prime}}^{2}-g_{A t_{L} t_{R}^{\prime}}^{2}-g_{A t_{L}^{\prime} t_{R}}^{2}+g_{h h t t} g_{A A t t}+g_{h h t^{\prime} t^{\prime}} g_{A A t^{\prime} t^{\prime}} \\
& =\frac{y^{2}}{2}\left(\frac{G^{\prime \prime}}{G}\right)^{2} \frac{1}{2}\left(1-\cos ^{2} \theta\right)\left(3 \cos ^{2} \theta-2\right) .
\end{aligned}
$$

Here we used the orthogonality relations among the mixing angles $s_{L, R}^{t}\left(c_{L, R}^{t}\right)$ which follows from the diagonalization of the fermion mass matrix in Eq. 52 with the rotation matrices in Eq. 555 :

$$
s_{L}^{t} s_{R}^{t} \cos \theta\left(\frac{G^{\prime \prime}}{G}\right)=\left(c_{L}^{t} \sin \theta-s_{L}^{t} \cos \theta\right) c_{R}^{t}, \quad c_{L}^{t} c_{R}^{t} \cos \theta\left(\frac{G^{\prime \prime}}{G}\right)=\left(s_{L}^{t} \sin \theta+c_{L}^{t} \cos \theta\right) s_{R}^{t}
$$

Thus we have Eq.79, and the associated formula for $A_{t}^{0}$ :

$$
\begin{aligned}
\left.\delta m_{h_{t}^{0}}^{2}\right|^{t, t^{\prime}} & =\frac{N_{c}}{8 \pi^{2}} \frac{y^{2}}{2}\left(\frac{G^{\prime \prime}}{G}\right)^{2}\left(2 \cos ^{2} \theta-1\right) \Lambda_{\chi}^{2} \\
& =\frac{N_{c}}{8 \pi^{2}}\left(\frac{\sqrt{2} m_{t}}{v_{\mathrm{EW}}}\right)^{2}\left(\frac{2 \cos ^{2} \theta-1}{\cos ^{2} \theta}\right) \Lambda_{\chi}^{2}\left[1+\mathcal{O}\left(\left(\frac{G^{\prime \prime}}{G}\right)^{2}\right)\right] \\
& =\text { Last term in Eq. }(79), \\
\left.\delta m_{A_{t}^{0}}^{2}\right|^{t, t^{\prime}} & =\frac{N_{c}}{8 \pi^{2}} \frac{y^{2}}{2}\left(\frac{G^{\prime \prime}}{G}\right)^{2} \frac{1}{2}\left(1-\cos ^{2} \theta\right)\left(3 \cos ^{2} \theta-2\right) \Lambda_{\chi}^{2} \\
& =\frac{N_{c}}{8 \pi^{2}}\left(\frac{\sqrt{2} m_{t}}{v_{\mathrm{EW}}}\right)^{2} \frac{\left(1-\cos ^{2} \theta\right)\left(3 \cos ^{2} \theta-2\right)}{2 \cos ^{2} \theta} \Lambda_{\chi}^{2}\left[1+\mathcal{O}\left(\left(\frac{G^{\prime \prime}}{G}\right)^{2}\right)\right],
\end{aligned}
$$

where the first lines in Eqs. (B20) and (B21) are exact results without further higher order corrections in $G^{\prime \prime} / G$ and in the second lines in Eqs. B20) and (B21) we used $m_{t}^{2}=\left(y^{2} f^{2} / 2\right) \sin ^{2} \theta \cos ^{2} \theta\left(G^{\prime \prime} / G\right)^{2}\left[1+\mathcal{O}\left(\left(G^{\prime \prime} / G\right)^{2}\right)\right]$ which can be derived from Eq.(54) with Eq. (63) and $v_{\mathrm{EW}}=f \sin \theta$. As noted around Eq. (79), from Eq.(B20), we see that the perturbative $G^{\prime \prime} / G$ corrections contribute to the tHiggs mass at the order of $\mathcal{O}\left(\left(G^{\prime \prime} / G\right)^{2}\right)$, no correction of $\mathcal{O}\left(G^{\prime \prime} / G\right)$, in accord with the Dashen formula Eq.(51), as well as the $A_{t}^{0}$ mass in Eq.(B21).

In the limit where $m_{t} \ll m_{t^{\prime}} \sim \Lambda_{\chi}$, the $t^{\prime}$-quark may be integrated out to induce the effective $h_{t}^{0}-h_{t}^{0}-t-t$ and $A_{t}^{0}-A_{t}^{0}-t$-t-couplings,

$$
g_{h h t t}^{\prime}=\frac{y}{2 \sqrt{2} f} \frac{g_{h t_{L} t_{R}^{\prime}} g_{h t_{L}^{\prime} t_{R}}}{g_{h h t^{\prime} t^{\prime}}} \quad, \quad g_{A A t t}^{\prime}=\frac{-y}{2 \sqrt{2} f} \frac{g_{A t_{L} t_{R}^{\prime}} g_{A t_{L}^{\prime} t_{R}}}{g_{h h t^{\prime} t^{\prime}}} .
$$


In that case, $\mathcal{C}_{h, A}$ in Eq. B16 become, up to order of $\left(G^{\prime \prime} / G\right)^{2}$,

$$
\begin{aligned}
\mathcal{C}_{h} & =-y_{h t t}^{2}+g_{h h t t}^{2}+g_{h h t t} g_{h h t t}^{\prime} \\
& =\frac{y^{2}}{2}\left(\frac{G^{\prime \prime}}{G}\right)^{2}\left(-1+6 \cos ^{2} \theta-6 \cos ^{4} \theta\right)\left[1+\mathcal{O}\left(\left(\frac{G^{\prime \prime}}{G}\right)^{2}\right)\right], \\
\mathcal{C}_{A} & =-y_{A t t}^{2}+g_{A A t t}^{2}+g_{h h t t} g_{A A t t}^{\prime} \\
& =\frac{y^{2}}{2}\left(\frac{G^{\prime \prime}}{G}\right)^{2} \frac{1}{2}\left(1-\cos ^{2} \theta\right)\left(-2+7 \cos ^{2} \theta-6 \cos ^{4} \theta\right)\left[1+\mathcal{O}\left(\left(\frac{G^{\prime \prime}}{G}\right)^{2}\right)\right] .
\end{aligned}
$$

Thus we find Eq. 80 and the associated result for $A_{t}^{0}$ :

$$
\begin{aligned}
\left.\delta m_{h_{t}^{0}}^{2}\right|^{t} & =\frac{N_{c}}{8 \pi^{2}} \frac{y^{2}}{2}\left(\frac{G^{\prime \prime}}{G}\right)^{2}\left(-1+6 \cos ^{2} \theta-6 \cos ^{4} \theta\right) m_{t^{\prime}}^{2}\left[1+\mathcal{O}\left(\left(\frac{G^{\prime \prime}}{G}\right)^{2}\right)\right] \\
& =-\frac{3}{8 \pi^{2}}\left(\frac{\sqrt{2} m_{t}}{v_{\mathrm{EW}}}\right)^{2} \frac{1-6 \cos ^{2} \theta+6 \cos ^{4} \theta}{\cos ^{2} \theta} m_{t^{\prime}}^{2}\left[1+\mathcal{O}\left(\left(\frac{G^{\prime \prime}}{G}\right)^{2}\right)\right] \\
& =\text { Last term in Eq. } \mathrm{E00}, \\
\left.\delta m_{A_{t}^{0}}^{2}\right|^{t} & =\frac{N_{c}}{8 \pi^{2}} \frac{y^{2}}{2}\left(\frac{G^{\prime \prime}}{G}\right)^{2} \frac{1}{2}\left(1-\cos ^{2} \theta\right)\left(-2+7 \cos ^{2} \theta-6 \cos ^{4} \theta\right) m_{t^{\prime}}^{2}\left[1+\mathcal{O}\left(\left(\frac{G^{\prime \prime}}{G}\right)^{2}\right)\right] \\
& =-\frac{3}{8 \pi^{2}}\left(\frac{\sqrt{2} m_{t}}{v_{\mathrm{EW}}}\right)^{2} \frac{\left(1-\cos ^{2} \theta\right)\left(2-7 \cos ^{2} \theta+6 \cos ^{4} \theta\right)}{2 \cos ^{2} \theta} m_{t^{\prime}}^{2}\left[1+\mathcal{O}\left(\left(\frac{G^{\prime \prime}}{G}\right)^{2}\right)\right] .
\end{aligned}
$$

[1] G. Aad et al. (ATLAS), Phys.Lett. B716, 1 (2012), 1207.7214.

[2] S. Chatrchyan et al. (CMS), Phys.Lett. B716, 30 (2012), 1207.7235.

[3] K. Yamawaki, M. Bando, and K.-i. Matumoto, Phys.Rev.Lett. 56, 1335 (1986).

[4] M. Bando, K.-i. Matumoto, and K. Yamawaki, Phys.Lett. B178, 308 (1986).

[5] S. Matsuzaki and K. Yamawaki, Phys.Lett. B719, 378 (2013), 1207.5911.

[6] S. Matsuzaki and K. Yamawaki, Phys.Rev. D86, 115004 (2012), 1209.2017.

[7] Y. Aoki, T. Aoyama, M. Kurachi, T. Maskawa, K. Miura, K.-i. Nagai, H. Ohki, E. Rinaldi, A. Shibata, K. Yamawaki and T. Yamazaki (2013), 1309.0711.

[8] V. Miransky and K. Yamawaki, Mod.Phys.Lett. A4, 129 (1989).

[9] K. Matumoto, Prog.Theor.Phys. 81, 277 (1989).

[10] T. Appelquist, M. Einhorn, T. Takeuchi, and L. Wijewardhana, Phys.Lett. B220, 223 (1989).

[11] V. Miransky, M. Tanabashi, and K. Yamawaki, Phys.Lett. B221, 177 (1989).

[12] V. Miransky, M. Tanabashi, and K. Yamawaki, Mod.Phys.Lett. A4, 1043 (1989).

[13] Y. Nambu (1989), EFI-89-08.

[14] W. Marciano, Phys.Rev.Lett. 62, 2793 (1989).

[15] W. J. Marciano, Phys.Rev. D41, 219 (1990).

[16] W. A. Bardeen, C. T. Hill, and M. Lindner, Phys.Rev. D41, 1647 (1990).

[17] K.-i. Kondo, H. Mino, and K. Yamawaki, Phys.Rev. D39, 2430 (1989).

[18] T. Appelquist, M. Soldate, T. Takeuchi, and L. Wijewardhana (1988), YCTP-P19-88.

[19] H. Pagels and S. Stokar, Phys.Rev. D20, 2947 (1979).

[20] J. Beringer et al. (Particle Data Group), Phys.Rev. D86, 010001 (2012).

[21] B. A. Dobrescu and C. T. Hill, Phys.Rev.Lett. 81, 2634 (1998), hep-ph/9712319.

[22] R. S. Chivukula, B. A. Dobrescu, H. Georgi, and C. T. Hill, Phys.Rev. D59, 075003 (1999), hep-ph/9809470.

[23] H.-J. He, C. T. Hill, and T. M. Tait, Phys.Rev. D65, 055006 (2002), hep-ph/0108041.

[24] H. S. Fukano and K. Tuominen, Phys.Rev. D85, 095025 (2012), 1202.6296.

[25] H. S. Fukano and K. Tuominen (2012), 1210.6756.

[26] The ATLAS collaboration, Tech. Rep. ATLAS-CONF-2013-034, CERN, Geneva (2013).

[27] The CMS collaboration, Tech. Rep. CMS-PAS-HIG-13-005, CERN, Geneva (2013).

[28] R. F. Dashen, Phys.Rev. 183, 1245 (1969).

[29] G. 't Hooft, NATO Adv.Study Inst.Ser.B Phys. 59, 135 (1980).

[30] N. Arkani-Hamed, A. Cohen, E. Katz, A. Nelson, T. Gregoire, et al., JHEP 0208, 021 (2002), hep-ph/0206020. 
[31] N. Arkani-Hamed, A. Cohen, E. Katz, and A. Nelson, JHEP 0207, 034 (2002), hep-ph/0206021.

[32] H.-C. Cheng and I. Low, JHEP 0309, 051 (2003), hep-ph/0308199.

[33] H.-C. Cheng and I. Low, JHEP 0408, 061 (2004), hep-ph/0405243.

[34] The ATLAS collaboration (ATLAS Collaboration) (2013), ATLAS-CONF-2013-034, ATLAS-COM-CONF-2013-035.

[35] M. E. Peskin (2012), 1207.2516.

[36] M. E. Peskin and T. Takeuchi, Phys.Rev.Lett. 65, 964 (1990).

[37] M. E. Peskin and T. Takeuchi, Phys.Rev. D46, 381 (1992).

[38] L. Lavoura and J. P. Silva, Phys.Rev. D47, 2046 (1993).

[39] M. Ciuchini, E. Franco, S. Mishima, and L. Silvestrini, JHEP 1308, 106 (2013), 1306.4644.

[40] The ATLAS collaboration (2013), ATLAS-CONF-2013-018, ATLAS-COM-CONF-2013-024.

[41] The ATLAS collaboration (2013), ATLAS-CONF-2013-056.

[42] The ATLAS collaboration (2013), ATLAS-CONF-2013-060, ATLAS-COM-CONF-2013-066.

[43] The CMS Collaboration (2013), CMS-PAS-B2G-12-015. 